\title{
Factores asociados al rendimiento académico tomando en cuenta el nivel socioeconómico: Estudio de regresión múltiple en estudiantes universitarios
}

\author{
Factors Associated with Academic Performance Including Socioeconomic Level: a \\ Multiple Regression Study of University Students
}

\author{
Guiselle María Garbanzo Vargas ${ }^{1}$ \\ Universidad de Costa Rica \\ Escuela de Administración Educativa \\ San José, Costa Rica \\ gmgarban@gmail.com
}

\begin{abstract}
Recibido 15 de julio de 2013 • Corregido 9 de octubre de 2013 • Aceptado 1 de diciembre de 2013
Resumen. La autora sistematiza una investigación cuantitativa sobre los factores asociados al rendimiento académico desde el nivel socioeconómico en estudiantes universitarios en la Universidad de Costa Rica; analiza, mediante modelos de regresión múltiple, los factores asociados al rendimiento académico en estudiantes que por su condición socioeconómica se les asignó la máxima categoría de beca y estudiantes que no solicitaron beca por esta condición de carreras de alta y baja demanda de la Universidad de Costa Rica. Se ofrece un marco explicativo sobre el tema, así como insumos para la gestión de la educación superior pública. Se evidencia entre los principales hallazgos que el rendimiento académico es un fenómeno altamente complejo y multicausal, así como se resaltan los principales factores institucionales, pedagógicos y psicosociales que se asocian al rendimiento académico tomando en cuenta el componente socioeconómico.
\end{abstract}

Palabras claves. Rendimiento académico, educación superior, rendimiento académico en estudiantes universitarios, rendimiento académico y condición socioeconómica, Universidad de Costa Rica.

Doctora en Educación de la Universidad de Costa Rica con pasantía de investigación en la Universidad de Salamanca, España, magister en Administración Educativa de la Universidad de Costa Rica, licenciada en Ciencias de la Educación con énfasis en Orientación de la Universidad de Costa Rica, licenciada en Administración con énfasis en Recursos Humanos de la Universidad Nacional y bachiller en Ciencias de la Educación con énfasis en Orientación de la Universidad de Costa Rica. Ha realizado pasantías académicas en la Universidad de Helsinki, Finlandia; Universidad de Murcia, España; Universidad de Montreal - Organización Universitaria Interamericana; también en la Unesco, Suiza, Ginebra. Además, ha participado en distintos congresos internacionales, entre ellos, en Brasil, México, Argentina y España. Ha publicado distintos artículos en el campo de la educación superior pública y de la administración de la educación en revistas nacionales e internacionales, ha impartido varias conferencias en el ámbito nacional e internacional, ha dirigido el II, III y IV Congreso Internacional de Administración de la Educación y ha sido directora de la Escuela de Administración Educativa, Facultad de Educación de la Universidad de Costa Rica en dos períodos. Actualmente es la directora del Posgrado en Ciencias de la Educación con énfasis en Administración Educativa, directora de la Revista Científica Gestión de la Educación. También es docente e investigadora en la Escuela de Administración Educativa de la Facultad de Educación de la Universidad de Costa Rica y coordina, para Centroamérica, el Curso de Gestión de Liderazgo Universitario. 


\begin{abstract}
The author systematically performed a quantitative research study of the factors associated with academic performance from a socioeconomic point of view in students from Universidad de Costa Rica. Using multiple regression models, the author analyzed factors associated with the academic performance of students awarded the highest level of scholarship because of their socioeconomic status and students not applying for a scholarship because they are enrolled in majors with high or low demand at Universidad de Costa Rica. This paper provides an explanatory framework on the subject, as well as inputs for the management of public higher education. One of the main findings indicates that academic performance is a multicausal and highly complex phenomenon. The paper also highlights the main institutional, pedagogical, and psychosocial factors associated with academic performance, taking into account the socioeconomic component.
\end{abstract}

Keywords. Academic performance, higher education, academic performance in college students, academic performance and socioeconomic status, University of Costa Rica.

En la presente época, la educación superior se enfrenta a una diversa y compleja oferta académica, la cual requiere de una cultura de innovación con rápidas y pertinentes reformas estructurales que le permita posicionarse en la desafiante sociedad del conocimiento y liderar procesos educativos de alto nivel. Estos desafíos la han hecho enfrentar cuestionamientos asociados a su calidad, donde el debate en torno al financiamiento no se ha hecho esperar. Los presupuestos estatales no necesariamente han asumido con correspondencia su crecimiento, lo que ha permitido que otras instancias de carácter privado tomen espacios en expansión. Este crecimiento se ha dado en forma importante en Latinoamérica, sin excepción; según datos del período 2000-2003, el 65\% de las universidades son privadas (Guni, 2009, citado por Vargas, 2010).

Esta expansión de la educación universitaria, aunada a la competitividad de la emergente sociedad del conocimiento del siglo XXI, trae, a su vez, importantes retos y dificultades en sus diferentes campos académicos, por los cuales las autoridades universitarias deben encauzar sus esfuerzos para ofrecer respuestas contundentes, sobre todo en los cuestionamientos referidos a la calidad.

El desempeño académico estudiantil se ubica entre los indicadores más destacados del desempeño de las instituciones educativas. Este se registra en el conjunto de materias que cursan los estudiantes en sus distintos planes de estudio y proporciona una guía válida de la eficiencia educativa en sus variados campos.

En general, en los estudios universitarios que buscan analizar los posibles factores asociados a los resultados del rendimiento académico, no es usual encontrar abordajes desde el nivel socioeconómico en estudiantes universitarios, a pesar de que este componente es gran valor para ofrecer insumos en la generación de políticas universitarias orientadas a la inclusión estudiantil.

El rendimiento académico ha sido estudiado por diferentes enfoques teóricos y metodológicos, en los que es frecuente encontrar el abordaje de este fenómeno, complejo y 
multicausal, mediante modelos de regresión lineal multinivel y modelos de ecuaciones lineales estructurales, por ejemplo. Usualmente, se aplican dimensiones teóricas que incluyen variables del orden personal, sociodemográfico, institucional, pedagógicas $\mathrm{y}$, en menor intensidad, se aborda según el nivel socioeconómico.

Específicamente, en la Universidad de Costa Rica, en los últimos años, esta Institución ha sido objeto de cuestionamientos en cuanto a su papel, en especial con las poblaciones de bajos niveles socioeconómicos. Se discute, además, hacia quiénes se dirigen los beneficios que ofrece $y$, si sus recursos y políticas se están orientando a dar oportunidades a este tipo de poblaciones (Ruiz, 2001). Lo anterior evidencia la importancia práctica que tienen, dentro de la Universidad de Costa Rica, investigaciones sobre el rendimiento académico en estudiantes desde su nivel socioeconómico.

En este contexto, se requiere tomar en cuenta que los nuevos desafíos de la educación superior -consecuencia de la globalización económica y de la sociedad de la información y el conocimiento- han expuesto la necesidad de revisar, constantemente, el rendimiento académico, según distintas variables. Por tanto, trabajar con estudiantes desde la condición socioeconómica es también una variable estratégica por investigar, sobre todo en el actual momento histórico la educación superior pública. Este elemento apoya el tema en estudio, cuyos resultados son referentes metodológicos y teóricos en esta materia, en cuanto a políticas institucionales orientadas al desarrollo del desempeño estudiantil. Esto, aunado a la particularidad desde la condición socioeconómica de su población de estudiantes, constituye un aporte para la Universidad de Costa Rica, sobre todo si se toma en cuenta que en la institución no se registran estudios con esta especificidad.

En cuanto al rendimiento académico de los estudiantes según su nivel socioeconómico, al no haber información disponible dentro de la Universidad de Costa Rica, se ofrece información generalizada con otro tipo de variables, tales como: institucionales, pedagógicas, sociales, demográficas y personales. Ante esta situación, surge el interés por analizar los factores asociados al rendimiento académico en estudiantes que por su condición socioeconómica se les asignó la máxima categoría de beca y estudiantes que no solicitaron beca por esta condición de carreras de alta y baja demanda de la Universidad de Costa Rica mediante modelos de regresión múltiple, buscando una aproximación a un modelo explicativo sobre el rendimiento académico.

Se busca poder determinar en qué medida los factores asociados al rendimiento académico difieren entre ambas poblaciones $y$, de esta forma, ofrecer insumos en este campo. Así se contribuye con las políticas institucionales en esta materia y, desde este ámbito, se aporta al fortalecimiento de la educación superior, medio importante en el desarrollo social (Freire, 2000; Rivero, 1999; Ruh, 1998). Esta información ofrecerá criterios más objetivos, apoyados en procesos científicos para la toma de decisiones de las políticas institucionales.

Es importante considerar que la educación superior pública, por su naturaleza social, posee un compromiso con su estudiantado y, en forma especial, con aquellas poblaciones estudiantiles con bajas condiciones socioeconómicas. 
Este interés asume relevancia en el sentido de que, en general, en la Universidad de Costa Rica se presentan diferencias entre las distintas unidades académicas, respecto al promedio de admisión de la Prueba de Aptitud Académica (PAA), debido a la demanda de las carreras. En algunos casos se ha mencionado que los estudiantes que poseen la máxima categoría de beca por condición socioeconómica tienden a ubicarse en carreras de baja demanda.

Precisamente, por esta demanda, en la sede "Rodrigo Facio" el Área de Salud presenta los promedios de admisión más altos, y el Área de Artes y Letras, los más bajos. Al respecto, un estudio realizado por Montero y Villalobos (2004a) indica que los estudiantes provenientes de colegios privados aventajan a los públicos respecto del promedio de admisión y el puntaje de la PAA.

\section{El rendimiento académico, nivel socioeconómico y el contexto universitario}

Los estudios sobre rendimiento académico en educación superior son escasos, comparados con otros niveles educativos. Asimismo, ha prevalecido un enfoque cuantitativo y han estado orientados al desarrollo de información de tipo económico (CEPAL, 2000). Las causas del rendimiento académico, en particular de estudiantes según niveles socioeconómicos, han sido poco exploradas.

El rendimiento académico en la educación superior pública, en estudiantes de bajos niveles socioeconómicos, en general, se aborda desde una perspectiva parcial, donde se hace referencia a poblaciones estudiantiles que ingresan en el sistema universitario por tipo de colegio y zona de procedencia; además, se apoya en estudios de corte estadístico (Rodríguez, Fita y Torrado, 2004). No son estudios que profundicen en las causas de un rendimiento académico en particular.

Es usual encontrar reportes sobre rendimiento académico referentes a la educación primaria y a la secundaria y, en menor medida, referidos al sistema universitario. Las informaciones ofrecidas tienen, en general, un carácter de rendición de cuentas del quehacer educativo, cuyo análisis se hace, la mayoría de veces, desde el punto de vista económico.

Entes gubernamentales y organismos internacionales como el Banco Mundial, citados por la CEPAL (2000), por ejemplo, han estudiado la educación y su relación con las condiciones sociales. Se incluye en estos estudios el tema del rendimiento académico; se considera fundamental la asociación entre las condiciones socioeconómicas y los diferentes resultados educativos y el campo de investigación en el que se incluye la educación superior. La educación se presenta como un motor fundamental del crecimiento económico y de la competitividad en el contexto de los nuevos mercados globalizados, y es percibida como uno de los principales medios de desarrollo social; asimismo, hay correlaciones significativas entre los niveles educativos y los niveles socioeconómicos adquiridos (Rivero, 1999).

Desde este punto de vista, no necesariamente la inversión en educación se podría traducir en desarrollo económico, no todas las políticas en educación pueden reducirse a transferencias 
de fondos a los poblaciones necesitadas socialmente, para que sean efectivas; es necesario complementarlas con otras políticas, entre ellas, aquellas que le lleguen a la raíz de los problemas del fracaso académico de los estudiantes de bajos niveles socioeconómicos (Arce, 2008 citado por Jiménez, Robles y Arce 2009).

Si bien es cierto, la educación se considera como importante en la superación de las condiciones socioeconómicas, en los últimos años, no es vista como el único medio para aventajar estas condiciones; existen otros factores vinculados, los cuales se relacionan con políticas socioeconómicas de orden mundial. No basta, por ejemplo, tener un título universitario para conseguir un buen empleo en la actualidad (Cañedo, 2008).

Estudios en América Latina demuestran que solo una minoría de la población de bajos niveles socioeconómicos de la región ingresa al sector educativo primario y logra mantenerse y acceder al nivel superior de la educación (CEPAL, 2000; Larrañaga, 1997; Ramos, 2000; UNESCO, 2002). Por esta razón, cobra relevancia investigar los factores asociados al rendimiento académico en estudiantes universitarios, según su nivel socioeconómico.

En este contexto, las universidades, especialmente en las últimas dos décadas, se encuentran ante nuevos desafíos, que han puesto en riesgo la calidad de la formación profesional. Se destacan como problemas actuales de la educación superior, la permanencia de los estudiantes de bajos niveles socioeconómicos, pues las políticas estatales han tendido a alejarse de un enfoque benefactor, para dar paso a la liberación de la economía (CEPAL, 2000). En este escenario, también es de interés profundizar en el tema de los factores asociados al rendimiento académico en estudiantes de bajos niveles socioeconómicos y poder, de esta forma, ofrecer un marco explicativo que apoye las políticas educativas universitarias.

\section{Rendimiento académico}

El rendimiento académico impacta no solo a nivel institucional, en lo referente al uso de los recursos, proyección externa-social, sino que también impacta en lo personal. Desde la perspectiva del estudiante, el rendimiento académico incide en sus esfuerzos, aspiraciones y proyectos personales, entre otros.

Son muchas las causas asociadas al rendimiento académico, por lo que se le considera un fenómeno multicausado; es decir, hay una tendencia a relacionar ciertas causas con el rendimiento académico, pero no se pueden generalizar a diferentes poblaciones.

Conceptualizar el rendimiento académico es difícil, debido a su multicausalidad y complejidad, ya que involucra factores como: actitudes, hábitos, carácter del personal docente, metodologías, formación profesional, ambiente familiar, organización del sistema educativo y condición socioeconómica, entre otros aspectos sociales, económicos y psicológicos (Valverde, 2005). En lo que parece haber consenso es que múltiples factores afectan el rendimiento académico: personales, sociales, económicos e institucionales. Por esa razón, estos 
condicionantes no se pueden homogeneizar. Esos factores se agrupan en varias categorías: sociodemográficos, psicosociales, pedagógicos e institucionales; es usual encontrar indicadores de orden socioeconómicos dentro de los sociodemográficos.

El rendimiento académico es un conjunto de factores multicausuales que impactan el resultado académico; intervienen en él aspectos de orden sociodemográfico, psicosociales, pedagógicos, institucionales y socioeconómicos; entre ellos, elementos tan variados como: la motivación, la ansiedad, la autoestima, la percepción del clima académico, el entusiasmo, el personal docente, el sentido de propósito y demás (Montero y Villalobos, 2004b).

El rendimiento académico en estudiantes universitarios es un indicador trascendental para la evaluación de la calidad educativa, se le considera como el resultado de la unión de diferentes elementos que interactúan en el desempeño de la vida académica del estudiantado (De Miguel, Apocada, Arias, Escudero, Rodríguez y Vidal, 2002). Este resultado se hace manifiesto a través de las calificaciones alcanzadas por cada estudiante, por lo general en una evaluación cuantitativa. El valor de las calificaciones representa los logros alcanzados en el desempeño académico, producto de una serie de variables personales, académicas y sociales que interactúan entre sí y entre ellas (Rodríguez, Fita y Torrado, 2004). Al respecto, Tournon (1984), citado por Montero, Villalobos y Valverde (2007), se refieren a variables que intervienen en el rendimiento académico como las pedagógicas, institucionales, sociodemográficas y psicosociales.

Los factores institucionales hacen referencia a características estructurales y funcionales, tales como los horarios de las materias, aspectos con relación directa a la carrera y el ambiente institucional (Montero, Villalobos y Valverde 2007).

Los factores psicosociales se asocian al binomio de interrelaciones en que actúa la sociedad y el individuo. Entre estos se encuentran conexiones plasmadas entre la sociedad y la persona; toma en cuenta aspectos como la autoestima y su relación con el entorno académico; motivación; percepción en torno al clima académico desde la óptica estudiantil, y ansiedad. Esta última en la medida en que modifica el valor predictivo de variables como la motivación y la inteligencia, pues por sí misma no es una variable predictiva del rendimiento académico (Montero, Villalobos y Valverde 2007). La competencia cognitiva, ambiciones académicas del estudiante hacia lo que proyecta lograr y motivación hacia un buen desempeño también son elementos considerados entre los factores psicosociales.

Entre los factores pedagógicos, se encuentra la influencia del rol docente, relación entre alumno y docente, habilidad de la parte docente para comunicarse con el estudiantado, interés académico que el docente pueda establecer entre cada estudiante -importante aquí la accesibilidad que muestre el docente hacia sus estudiantes, que los valore como personas integrales- (Centra, 1970, en Latiesa, 1992, ambos citados por Montero, Villalobos y Valverde, 2007). Los recursos didácticos, métodos de evaluación y estrategias de enseñanza también son factores pedagógicos asociados al rendimiento académico. 
Dentro de los factores sociodemográficos se encuentran aspectos como el nivel económico del grupo familiar, entorno socieoecónomico y sociocultural, sexo, modalidad de colegio, nivel educativo del padre y de la madre. El componente cultural y social del ambiente familiar se considera favorecedor del desarrollo intelectual (Montero, Villalobos y Valverde, 2007). Por su lado, los del orden socioeconómico incluyen una serie de bienes o posesiones que el grupo familiar posee.

Importante tomar en cuenta que cada institución educativa determina criterios por considerar en la evaluación para ofrecer una estimación o definir el rendimiento académico de su población estudiantil. Usualmente se le conceptualiza como el promedio ponderado, dentro del cual se considera la nota académica obtenida en las distintas materias, su valor en créditos y el número de materias matriculadas. En este resultado académico también inciden factores institucionales, sociales y personales.

Es evidente que el rendimiento académico es multicausal, intervienen en él factores de diversa naturaleza, los cuales deben ser considerados para su análisis: factores psicosociales, sociodemográficos, pedagógicos, socioeconómicos e institucionales, que por si mismos interactúan, y también lo hacen entre ellos. De estas interacciones se alcanza un determinado resultado, razón por la cual su estimación se caracteriza por un alto nivel de complejidad.

\section{Conceptualización del nivel socioeconómico}

El nivel socioeconómico es considerado una variable demográfica, ante la cual distintas instituciones, países y momentos históricos utilizan una definición diferente en su forma de cálculo. Esta heterogeneidad conceptual dificulta que sus resultados necesariamente sean comparables entre distintas instancias, razón por la cual, para su análisis, cada investigación, institución y sociedad ocupa definir con certeza la construcción de esta variable demográfica.

Con el propósito de buscar un consenso en esta conceptualización, la Asociación Mexicana de Agencias de Investigación de Mercados (AMAl) concluyó el nivel socioeconómico "no puede ser una característica de un individuo, ni de un grupo de individuos, sino que debe ser una característica de un hogar" (González, 2000, p. 1). "Es un esfuerzo por cuantificar una característica cualitativa de hogares" (González, 2000, p. 4). Se le asignan medidas cuantitativas a un conjunto de características del hogar, que pueden ser cuantitativas o cualitativas.

Para estos efectos, el hogar no se comprende únicamente como la vivienda, es -más bien- un grupo de individuos que pueden estar o no relacionados consanguíneamente, que dentro de su convivencia comparten la misma alacena, de esta manera las personas heredan el nivel socioeconómico del hogar y no de ninguno de sus progenitores en forma individual (González, 2000).

Partiendo de una serie de características propias de la constitución de cada hogar, de las personas que lo conforman y del entorno en que interactúa, se obtiene el nivel socioeconómico 
del hogar. De las referentes al entorno, se obtiene el componente social. Las del orden económico incluyen características del hogar, de las personas que lo integran, por ejemplo, posesiones. Dentro de las del entorno, características de la interacción, centros educativos a los que asiste, clubes, actividades culturales, entre otras (González, 2000).

El nivel socioeconómico toma en cuenta características cuantitativas y cualitativas. Solo se pertenece a un nivel socioeconómico, es un atributo del hogar compartido por sus miembros, su medida se recomienda a escala nacional. Importante tomar en cuenta que debido a las variables que interactúan en forma permanente, se proyecta a corto plazo.

Para una mejor comprensión conceptual en lo que respecta a los niveles socioeconómicos, es preciso ofrecer una definición de clases sociales y evitar cualquier ambigüedad que pueda darse. Las clases sociales son un "conjunto de personas con ciertas características que las hacen compartir una posición en la vida económica de un país. Esta posición está determinada por los modos en que las personas y familias participan en la organización reproductiva" (Programa Estado de la Nación en Desarrollo Humano Sostenible, 2009, p. 339).

Sustentado en este mismo Informe del Programa Estado de la Nación en Desarrollo Humano (2009), las clases sociales vienen a ser el medio con el cual se articula un análisis de la desigualdad con las condiciones que deben desarrollarse y las oportunidades a las cuales se desea acceder. Las clases sociales son un criterio significativo para abordar el tema de la desigualdad; además, el tema de las clases sociales proporciona una perspectiva analítica en la generación del conocimiento en el abordaje del tema del desarrollo humano y ofrece importantes insumos en políticas públicas.

La categorización por clases sociales de los diferentes grupos es considerada como uno de los criterios de mayor relevancia para captar las desigualdades sociales de una sociedad determinada. El estudio de las clases sociales ha tenido, a lo largo de la historia, distintas posiciones teóricas, destacándose dos grandes, como son la marxista y la weberiana. La primera reconoce dos clases sociales fundamentales, la burguesía y el proletariado. La de Weber se caracterizó por la descripción de tres jerarquías de desigualdad: económica, social y política. Conceptualmente, es un tema difícil de consensuar; sin embargo, sí hay consenso en que implica una visión de la estructura de la organización de la sociedad, donde su parámetro son las formas que poseen las personas para insertarse y relacionarse entre sí en el campo económico (Programa Estado de la Nación en Desarrollo Humano Sostenible, 2009).

En lo que a clase social se refiere, la sociedad costarricense ha dejado de ser mucho menos obrera y pequeña propietaria, y menos rural y más urbana que antes. Ha experimentado un cambio radical en este campo, de manera que modificó su estructura de clases sociales; además, se caracteriza por poseer, en la actualidad, una distribución de ingresos más desigual que hace veinte años (Programa Estado de la Nación en Desarrollo Humano Sostenible, 2009).

En Costa Rica, el Programa Estado de la Nación en Desarrollo Humano Sostenible (2009), sustentado en distintos enfoques teóricos y estudios previos, realizó una propuesta de estructura 
de clases sociales, dando como resultado seis categorías: clase alta, medianos empresarios y expertos, clases intermedias, pequeños propietarios, obreros agrícolas, obreros industriales, obreros en servicio y otros trabajadores.

En el contexto costarricense, los niveles socioeconómicos son considerados como una característica del hogar que comparten sus miembros, se establece por una serie de variables relacionadas específicamente por la capacidad de consumo que posee un grupo familiar. Importante indicar que la Universidad de Costa Rica no clasifica sus estudiantes según la estratificación social que utiliza la sociedad costarricense, ella establece sus propios indicadores.

\section{Nivel socioeconómico y rendimiento académico en la educación superior}

Dentro de las variables utilizadas de corte socioeconómico, se ha considerado en la educación superior el trabajo del alumno, el nivel educativo de los padres y el nivel económico. Al respecto, (Armenta, Pacheco y Pineda, 2008) consideran que, a mayor nivel económico, mejor es el rendimiento académico, específicamente; en el sentido de que cuando el estudiante universitario trabaja, su rendimiento académico baja.

Por su parte, Plasencia, Vizconde, Ruiz, Araujo y Salazar y (2008), al desarrollar el tema sobre la influencia de los factores socioeconómicos en el desempeño académico universitario, consideran que sí hay influencia entre los factores socioeconómicos y el rendimiento académico, pues en los alumnos de escasos recursos económicos se da un rendimiento académico bajo, que en algunos casos, origina el abandono de los estudios. Se tomaron en cuenta en esta asociación la posesión de vivienda propia con servicios básicos en su hogar de origen, características de estas viviendas como el hacinamiento y aspectos estructurales, el origen del sostén económico de los estudiantes y el hecho de que el estudiante labore o no lo haga, además, de estudiar. También, se consideró el presupuesto en alimentación y disponibilidad para cubrir los costos de los estudios universitarios. Interesa, en este campo, la necesidad de que la universidad como institución se preocupe por ofrecer condiciones institucionales en la vida académica de estudiantes de bajos niveles socioeconómicos, de manera que incida en un mejor desempeño académico.

No obstante, son variados los casos en que se afirma que los aspectos de orden socioeconómico influyen de manera significativa en el rendimiento académico del estudiantado. Esta asociación favorece mayores o menores posibilidades en los recursos didácticos requeridos para un óptimo rendimiento académico. Definitivamente, información asociada con el nivel socioeconómico les permite a las universidades tomar medidas para favorecer, de esta forma, el rendimiento académico (Pirrón, Rojas y Arzola, 2009).

Ramos (2000), al referirse a las condiciones contextuales que se asocian a la permanencia académica de los alumnos de bajos niveles socioeconómicos en la educación y especialmente en el nivel superior, considera que es fundamental para el estudiante requerir de condiciones materiales mínimas para un adecuado desempeño académico y que este nivel superior de la educación, específicamente el público, por su propia naturaleza requiere de una revisión 
constante de las posibilidades que le ofrece, particularmente, a estudiantes de bajos niveles socioeconómicos para un adecuado rendimiento académico.

A manera de síntesis, en este apartado se puede decir que el bajo nivel educativo tiende a asociarse con niveles socioeconómicos bajos, las personas de estos niveles se asocian con la categoría de pobres y, al respecto, la población en edad escolar de hogares pobres reproduce similares condiciones socioeconómicas en las que viven los padres ${ }^{2}$. Dicho fenómeno es bastante determinado por el bajo nivel educativo de los padres y el menor nivel de ingreso de los hogares, según lo demuestra Ramos (2000)․․

A mayor educación del padre, mayores posibilidades de logro educativo en los hijos. En los padres que registraron no tener educación, solo se da un $6 \%$ de hijos en edad normativa $y$, al contrario, se eleva al $65 \%$ de hijos con posibilidades de logro educativo. Además, de comprobarse que la variable educación de los padres es definitiva en el resultado educativo de los hijos, también lo es la variable del ingreso de los hogares; ambas son influyentes en la determinación del logro educativo de los hijos (Ramos, 2000).

Los hogares con bajo logro educativo son las principales fuentes de generación de futuros pobres; personas cuyos hogares son caracterizados con bajos niveles socioeconómicos. El fenómeno del atraso educativo no es exclusivo de un determinado tipo de hogar, ya que también los hogares de altos niveles socioeconómicos podrían estar generando, también, futuros pobres, por poseer también porcentajes, aunque menor, de hijos con bajo logro educativo (Ramos, 2000).

Las desigualdades de origen social impactan en el desarrollo académico de manera significativa. La relación entre estudiantes provenientes de hogares de bajos niveles socioeconómicos y un bajo nivel educativo se da con pocas posibilidades de alcanzar altos niveles de escolaridad. En un 58\% y $60 \%$ es la probabilidad de que hijos e hijas multipliquen el nivel educativo de sus padres.

\section{Consideraciones metodológicas del estudio}

La presente investigación aplicó técnicas de recolección y análisis cuantitativo, utilizó un cuestionario y relaciona el rendimiento académico como variable dependiente con una serie de variables independientes, mediante el uso de dos modelos de regresión múltiple; un modelo para estudiantes de carreras de alta demanda, con estudiantes con la máxima categoría de beca

2 El estudio realizado en Perú por el Instituto Nacional de Estadística, con datos de la ENAHO (Encuesta Nacional de Hogares) 1998, del segundo trimestre, y en parte se sostiene en resultados de investigaciones efectuadas por el CEPAL y el Banco Mundial sobre la importancia de la educación para el desarrollo.

3 El estudio permitió seleccionar variables predictivas sobre el logro educativo para ser utilizadas como instrumentos de política educativa. Se construyó la variable dependiente "logro educativo", que representa a los hijos con años de estudio aprobados en relación con la edad normativa. Como variables independientes (contextuales) imputables al hogar, el gasto social del Estado y otros factores sociales. 
por condición socioeconómica y estudiantes que no solicitaron beca por esta condición, y el otro, para la población de carreras de baja demanda, con estudiantes con la máxima categoría de beca por condición socioeconómica y estudiantes que no solicitaron beca por esta condición.

En un modelo de regresión, se elabora una ecuación en la que la variable dependiente, en este caso, el rendimiento académico $(\mathrm{Y})$, es la que interesa predecir; se percibe como una función lineal de varias variables independientes o predictoras (nivel socioeconómico y otras) (X1,X2,X3....Xn). A esta función se le conoce como ecuación de regresión.

Se parte del supuesto de que el valor que se observa en la variable dependiente, es decir, en el rendimiento académico, es el producto de la relación lineal de otras variables. Las variables independientes son los factores relevantes que se estudiaron. De esta forma, se analizó si las variables independientes permiten predecir y con qué nivel de precisión el comportamiento de la variable que interesa (rendimiento académico). Este tipo de técnica estadística posibilita analizar e interpretar la intensidad de la asociación que interesa investigar entre las variables involucradas, el determinar el grado de relación entre ellas y controlar el efecto de la variable independiente en el modelo.

Se recurrió a examinar la magnitud de los coeficientes de regresión para poder estimar la intensidad e importancia relativa de las variables independientes en lo que a predicción de la variable dependiente se refiere (rendimiento académico). De este modo, se podría determinar si existe o no una relación funcional entre las variables (Johnson, 1988).

Para poder evaluar la calidad del modelo de regresión planteado, se utiliza como una medida de bondad de ajuste el coeficiente de determinación, el cual, según Martínez (2005, p. 320), es "una medida de dispersión relativa para la ecuación de regresión, comparando la misma con la varianza total de $Y$ ". El coeficiente de determinación se interpreta "como la proporción o porcentaje de variación total en $Y$ respecto de su medida, que es explicada por el modelo de regresión" Martínez (2005, p. 320).

"Un $R^{2}$ igual a 1 significa un ajuste lineal perfecto, ya que STC = SEC, esto es, la variación total de la variable $Y^{\prime \prime}$ (Martínez, 2005, p. 321). Además de los coeficientes de regresión, se utiliza otra medida coeficiente de regresión parcial estandarizado (coeficiente Beta); estos coeficientes permiten medir la importancia relativa de cada una de las variables predictoras dentro del modelo, y se obtiene estandarizando las variables originales.

Estos coeficientes se consideran con valores relevantes cuando son superiores a 0,10. Es posible compararlos de manera directa de forma válida, debido a que la estandarización llega a eliminar el efecto derivado de las diferentes unidades de medida de las respectivas variables. El estudio se llevó a cabo en distintas unidades académicas, de manera que permita un adecuado control de las variables.

\section{Criterio para seleccionar la población del estudio}

La selección de los estudiantes se hizo utilizando una cohorte de ingreso del 2003, cuya mayoría, en el momento del estudio, estaban entre el último, penúltimo año de carrera o graduados. 
Los criterios para seleccionar al estudiantado, según el nivel socioeconómico, fueron los de la Oficina de Atención Socioeconómica de la Universidad de Costa Rica, dentro de los cuales, en el momento del estudio se consideraban como estudiantes con la máxima categoría de beca por su condición socioeconómica a aquellos que, por esta condición, solicitaron beca y se les otorgó la máxima categoría que la Universidad asigna de ayuda socioeconómica, conocida como beca once. Por su lado, los estudiantes que no solicitaron beca, son los que no necesitaron ningún tipo de ayuda socioeconómica. La máxima categoría de ayuda socioeconómica que ofrece la Universidad de Costa Rica, se asigna utilizando las siguientes variables: ingreso per cápita, colegio de procedencia, imponible al ruedo e imponible a la propiedad. Estas variables se actualizan mediante una serie de indicadores como inflación, línea de pobreza, ingreso per cápita por quintil, aranceles de cobro, índice de precios al consumidor, pensión Régimen Invalidez, Vejez y Muerte, pensión de Régimen no contributivo y salarios mínimos (Universidad de Costa Rica, Oficina de Becas y Atención Socioeconómica, 2009).

\section{Operacionalización e instrumentalización de los factores asociados al rendimiento académico}

Algunas de las variables independientes, se construyeron, mediante escalas, en total seis escalas. También se construyó el índice de nivel socioeconómico.

Estas escalas fluctúan según la cantidad de ítems, y todos ellos son indicadores de una misma naturaleza conceptual, miden el constructo teórico al que pertenece la escala y tiene carácter unidimensional. La escala es una medida compuesta mediante la cual se relacionan variables de una misma naturaleza, se usa con mayor frecuencia para medir constructos e implica un marco de referencia teórico. Para evaluar si el conjunto de ítems cumple con el requisito de una escala, unidimensionalidad, se utilizó el análisis de factores, el cual es una técnica de la estadística multivariada que permite determinar si lo que se mide corresponde a una escala o índice, en caso de que la estructura factorial indique una dimensión, hay evidencia de que los ítems utilizados conforman una escala (Montero, 2008). Una vez demostrado que estos ítems miden solo una dimensión, se procedió a analizar la confiabilidad de estas escalas, por medio de Alfa de Cronbach y correlaciones de cada ítem con la escala.

\section{Variable dependiente: rendimiento académico}

Es el promedio ponderado acumulado del estudiante al momento del estudio. Se asume el rendimiento académico como el conjunto de factores multicausales que impactan en el resultado académico, donde intervienen factores de orden socioedemográfico, psicosociales, pedagógicos e institucionales (Montero y Villalobos 2004b). El rendimiento académico es el resultado de la unión de diferentes factores que intervienen en la vida académica estudiantil. Es el valor atribuido al logro del estudiante en su desempeño académico que se muestra mediante las calificaciones obtenidas. Por lo general, se acompaña de una evaluación cuantitativa del 
aprovechamiento del estudiante, puede ser o no satisfactorio, o implicar deserción o éxito académico (Pérez, Ramón y Sánchez, 2000; Vélez y Roa, 2005). En el campo operativo, son los factores que indicen en el resultado académico de estudiantes de carreras de alta y baja demanda de estudiantes con la máxima categoría de beca por su condición socioeconómica y estudiantes no solicitantes de beca por esta condición, donde intervienen factores de orden socioedemográfico, psicosociales, pedagógicos e institucionales.

\section{Componente nivel socioeconómico, criterios metodológicos para la selección de los estudiantes}

El nivel socioeconómico se trabaja en dos componentes: estudiantes con la máxima categoría de beca que por su condición socioeconómica se les otorgó esta categoría, denominados en el estudio como "estudiantes con la máxima categoría de beca" y estudiantes que no solicitaron beca por esta condición socioeconómica, denominados en el estudio como "estudiantes que no solicitaron beca" de carreras de alta demanda y baja demanda. La designación de la agrupación de estos estudiantes se hace según los parámetros que la Oficina de Atención Socioeconómica de la Universidad de Costa Rica posee para asignar la máxima ayuda socioeconómica que por esta condición requiera el estudiante. La Universidad de Costa Rica utiliza distintas categorías de becas por condición socioeconómica, que van de 0 a 11, donde la beca $11^{4}$ corresponde a la máxima categoría que se asigna a los estudiantes de niveles socioeconómicos más bajos.

Por los objetivos del presente estudio, a pesar de que existen en la Universidad de Costa Rica categorías de beca por condición socioeconómica que va de 1 a 11, no se tomaron en cuenta los de beca 1 a la 10, ya que interesa trabajar con aquellos estudiantes que muestren la mayor necesidad socioeconómica y poderlos contrastar con los que no solicitaron beca.

\section{Criterios metodológicos para la selección de las unidades académicas}

Mediante información proporcionada por la Vicerrectoría de Vida Estudiantil y la Oficina de Registro de la Universidad de Costa Rica, con datos de 2003, se levantó un listado sobre las unidades académicas de baja demanda, mediana y alta demanda del año de la cohorte, es decir, del 2003, en que la población en estudio ingresó. La demanda de las carreras se determinó con base en el número de las solicitudes de ingreso: se indicaron como carreras de alta demanda aquellas con 400 solicitudes o más; de demanda media, las que tuvieron 100 a 399 solicitudes y como de baja demanda, las carreras con menos de 100 solicitudes. Esta información se ilustra en tablas 1 y 2 :

$4 \quad$ La beca 11 es la máxima categoría que se otorga por condición socioeconómica baja, según los indicadores que para estos efectos establece la Oficina de Atención Socioeconómica de la Universidad de Costa Rica. 
URL: http://www.una.ac.cr/educare

CORREO: educare@una.cr

Tabla 1

Distribución de las unidades académicas seleccionadas

\begin{tabular}{|c|c|c|}
\hline Nivel económico & Alta demanda & Baja demanda \\
\hline Estudiantes que no solicitaron beca * & 1. Derecho & $\begin{array}{l}\text { 1. Artes Plásticas, énfasis Diseño Gráfico } \\
\text { 1. Tecnología de Alimentos } \\
\text { 2. Artes Dramáticas }\end{array}$ \\
\hline $\begin{array}{l}\text { Estudiantes con la máxima categoría } \\
\text { de beca }{ }^{* *}\end{array}$ & $\begin{array}{l}\text { 1. Dirección de Empresas } \\
\text { 2. Odontología } \\
\text { 3. Historia } \\
\text { 4. Fitotecnia } \\
\text { 5. Derecho }\end{array}$ & $\begin{array}{l}\text { 1. Economía Agrícola } \\
\text { 2. Archivística } \\
\text { 3. Artes Plásticas, énfasis Diseño Gráfico } \\
\text { 4. Geología } \\
\text { 5. Educación Especial } \\
\text { 6. Primaria } \\
\text { 7. Orientación } \\
\text { 8. Educación Física } \\
\text { 9. Geografía } \\
\text { 10. Asistente de Laboratorio }\end{array}$ \\
\hline
\end{tabular}

Nota. Corresponde al procesamiento estadístico del estudio.

Universidad de Costa Rica, Oficina de Registro (2003).

* $\sin$ beca / ** con beca 11

Tabla 2

Distribución de carreras de alta y baja demanda que agrupan la mayoría de estudiantes con la máxima categoría de beca y estudiantes que no solicitaron beca

\begin{tabular}{llll}
\hline \multicolumn{1}{c}{ Nivel económico } & \multicolumn{1}{c}{$\begin{array}{c}\text { Alta demanda } \\
\text { porcentaje de estudiantes } \\
\text { según beca }\end{array}$} & \multicolumn{1}{c}{$\begin{array}{c}\text { Baja demanda } \\
\text { porcentaje de estudiantes según beca }\end{array}$} \\
\hline $\begin{array}{l}\text { Carrera (s) que agrupó la } \\
\text { mayoría de estudiantes }\end{array}$ & 1. Derecho (93.71\%) & $\begin{array}{l}\text { 1. Artes Plásticas, énfasis Diseño Gráfico (87.10 \%) } \\
\text { que no solicitaron beca }\end{array}$ & $\begin{array}{l}\text { 2. } \\
\text { 3ecnología de Alimentos (88.89\%) }\end{array}$ \\
Carrera (s) que agrupó la & 1. Dirección de Empresas (7\%) & 1. Economía Agrícola (10.42\%) \\
mayoría de estudiantes & 2. Odontología (6.49\%) & 2. Archivística (18.18\%) \\
con la máxima categoría & 3. Historia (6.06\%) & 3. Artes Plásticas, énfasis Diseño Gráfico (9.68\%) \\
de beca. & 4. Fitotecnia (10\%) & 4. Geología (8.89\%) \\
& 5. Derecho (4.90\%) & 5. Educación Especial (11.11\%) \\
& & 6. Primaria (21.21\%) \\
& & 7. Orientación (15.79\%) \\
& & 8. Educación Física (9.68\%) \\
& & 9. Geografía (13.33\%) \\
& & 10. Asistente de Laboratorio (18.18\%) \\
\hline
\end{tabular}

Nota: Universidad de Costa Rica, Oficina de Registro (2003) y Universidad de Costa Rica. Oficina de Becas y Atención Socioeconómica (2009). 


\section{Caracterización de la muestra}

La muestra se conformó por 120 estudiantes que ingresaron a la Universidad de Costa Rica en el 2003, y de ellos seleccionaron 60 estudiantes con la máxima categoría de beca, distribuidos en carreras de alta demanda y baja demanda. Los otros 60 estudiantes se seleccionaron por considerarse estudiantes que no solicitaron beca, distribuidos también en carreras de alta demanda y baja demanda.

Se logró obtener información de 120 estudiantes, donde la edad promedio es de 23 años, en su mayoría provienen de colegio académicos, diurnos y públicos. La edad promedio de finalización de la secundaria y de inicio de la universidad es prácticamente la misma. La mayoría proviene de colegios públicos y diurnos, una pequeña población no asistió a educación preescolar. Respecto de la educación del padre y la madre, la universitaria completa es el nivel educativo que presenta mayor proporción: $44.2 \%$ el padre y $41.7 \%$ la madre. La mayoría de estudiantes no tiene económicamente a cargo ninguna persona. Cerca de la mitad de la población son estudiantes que laboran en época lectiva. Mediante el análisis de correlación para verificar cuán fuerte es la asociación entre la nota de admisión y el promedio ponderado, resultó tener este una correlación de 0.22; no es muy alta, pero sí es significativa a un 5\%, tiene una significancia de 0.02.

En la tabla 3 se presentan las diferencias entre los estudiantes de la población del estudio con la máxima categoría de beca, es decir, la beca 11 y estudiantes que no solicitaron beca, en este caso denominados sin beca, según variables independientes del estudio, lo que se hace mediante una tabla $2 \times 2$ :

Tabla 3

Características de estudiantes con beca 11 y sin beca en carreras de alta y baja demanda: variablescategóricas (valores absolutos)

\begin{tabular}{|c|c|c|c|c|c|c|}
\hline \multirow[b]{2}{*}{ Variable } & \multirow[b]{2}{*}{ Categorías } & \multicolumn{2}{|c|}{ Alta demanda } & \multicolumn{2}{|c|}{ Baja demanda } & \\
\hline & & Sin beca & Beca 11 & Sin beca & Beca 11 & \\
\hline \multirow[t]{3}{*}{ Sexo } & & 31 & 29 & 30 & 30 & \\
\hline & Masculino & 19 & 18 & 12 & 12 & \\
\hline & Femenino & 12 & 11 & 18 & 18 & \\
\hline \multirow[t]{3}{*}{ Modalidad de colegio } & & 31 & 29 & 30 & 30 & ** \\
\hline & Publico & 11 & 28 & 6 & 27 & \\
\hline & Privado o subvencionado & 20 & 1 & 24 & 3 & \\
\hline \multirow[t]{3}{*}{ Tipo de colegio } & & 31 & 29 & 30 & 30 & \\
\hline & Diurno & 30 & 28 & 30 & 27 & \\
\hline & Nocturno & 1 & 1 & 0 & 3 & \\
\hline
\end{tabular}

Continúa... 
URL: http://www.una.ac.cr/educare

CORREO: educare@una.cr

\begin{tabular}{|c|c|c|c|c|c|c|}
\hline \multirow[b]{2}{*}{ Variable } & \multirow[b]{2}{*}{ Categorías } & \multicolumn{2}{|c|}{ Alta demanda } & \multicolumn{2}{|c|}{ Baja demanda } & \\
\hline & & Sin beca & Beca 11 & Sin beca & Beca 11 & \\
\hline \multirow[t]{3}{*}{ Educación preescolar } & & 31 & 29 & 30 & 29 & * \\
\hline & Sí & 28 & 19 & 25 & 20 & \\
\hline & No & 3 & 10 & 5 & 9 & \\
\hline \multirow[t]{3}{*}{ Es la carrera que deseaba estudiar } & & 31 & 29 & 30 & 30 & \\
\hline & Sí & 26 & 23 & 24 & 20 & \\
\hline & No & 5 & 6 & 6 & 10 & \\
\hline \multirow[t]{3}{*}{ Lugar de residencia } & & 31 & 29 & 30 & 30 & * \\
\hline & En casa de su familia & 24 & 20 & 24 & 15 & \\
\hline & En otro lugar & 7 & 9 & 6 & 15 & \\
\hline \multicolumn{2}{|l|}{ Conversaciones sobre temas académicos en la familia } & 31 & 29 & 30 & 30 & * \\
\hline & Sí & 16 & 10 & 18 & 9 & \\
\hline & No & 15 & 19 & 12 & 21 & \\
\hline \multirow[t]{3}{*}{ La educación representa un valor importante } & & 31 & 29 & 30 & 30 & \\
\hline & Sí & 29 & 27 & 28 & 27 & \\
\hline & No & 2 & 2 & 2 & 3 & \\
\hline \multirow[t]{3}{*}{ Como considera que es la convivencia familiar } & & 31 & 29 & 30 & 30 & \\
\hline & Muy buena & 27 & 24 & 25 & 19 & \\
\hline & Regular o mala & 4 & 5 & 5 & 11 & \\
\hline \multirow[t]{3}{*}{ Vive con sus padres biológicos } & & 31 & 29 & 30 & 29 & \\
\hline & Sí & 26 & 24 & 24 & 19 & \\
\hline & No & 5 & 5 & 6 & 10 & \\
\hline \multirow[t]{3}{*}{ Como valora la relación con su padre } & & 29 & 21 & 23 & 20 & \\
\hline & Abierta al dialogo & 28 & 20 & 19 & 18 & \\
\hline & Impositiva y de temor & 1 & 1 & 4 & 2 & \\
\hline \multirow[t]{3}{*}{ Como valora la relación con su madre } & & 31 & 28 & 30 & 27 & \\
\hline & Abierta al dialogo & 31 & 28 & 28 & 26 & \\
\hline & Impositiva y de temor & 0 & 0 & 2 & 1 & \\
\hline \multicolumn{2}{|l|}{ Experimenta en la actualidad situaciones de violencia } & 31 & 29 & 30 & 30 & \\
\hline & Sí & 1 & 3 & 1 & 3 & \\
\hline & No & 30 & 26 & 29 & 27 & \\
\hline \multicolumn{2}{|l|}{ Experimentó en el pasado situaciones de violencia } & 31 & 29 & 30 & 30 & * \\
\hline & Sí & 4 & 7 & 3 & 11 & \\
\hline & No & 27 & 22 & 27 & 19 & \\
\hline
\end{tabular}

Notas: *Diferencias significativas en alguno de los grupos. ${ }^{*}$ Diferencias significativas en ambos grupos. Se utilizó la prueba estadística del coeficiente de contingencia, para probar diferencias. 


\section{Grupo de estudiantes de carreras de alta demanda}

\section{Correlaciones de variables}

\section{Variables categóricas}

En los modelos de regresión, la variable dependiente es el promedio ponderado. Al respecto, se hizo un análisis de correlaciones de las variables que se introdujeron en el modelo con el promedio ponderado.

Para medir la asociación entre las variables categóricas o dummy y el promedio ponderado, se calculó el estadístico Eta, el cual mide la asociación entre una variable métrica y una categórica; este estadístico varía de 0 a 1 (ver tabla 4). De las presentes en el modelo, se puede decir que las que tienen una mayor asociación con el promedio ponderado (por lo menos en este grupo) son: lugar de residencia en tiempo lectivo, situaciones de violencia familiar en el pasado, percepción de la convivencia familiar y la carrera que deseaba estudiar.

Respecto de la variable "situaciones de violencia familiar en el pasado", llama la atención que la violencia familiar en la actualidad no haya resultado como una variable muy asociada con el rendimiento académico, como sí lo hace la violencia familiar en el pasado, que resultó muy asociada. Podría pensarse que el estudiante, en caso de vivirla en la actualidad, no se sienta cómodo ofreciendo esta información.

El resto de las variables presentan una asociación muy baja con la variable dependiente. La siguiente tabla hace referencia a esta asociación.

Tabla 4

Asociación del promedio ponderado con las variables categóricas (estadístico de asociación Eta)

\begin{tabular}{|c|c|}
\hline Variables & Promedio ponderado \\
\hline Sexo $(1=$ hombre, $0=$ mujer $)$ & 0,20 \\
\hline Modalidad del colegio ( $1=$ público, $0=$ privado) & 0,16 \\
\hline Educación preescolar ( $1=$ sí asistió, $0=$ no asistió) & 0,16 \\
\hline Es la carrera que deseaba estudiar $(1=s i, 0=n o)$ & 0,21 \\
\hline $\begin{array}{l}\text { Lugar de residencia en tiempo lectivo ( } 1=\text { en casa de parientes, residencias universitarias, } \\
\text { apartamento, } 0=\text { en casa de su familia) }\end{array}$ & 0,38 \\
\hline Beca (1=asignación de beca 11, $0=\sin$ beca $)$ & 0,16 \\
\hline $\begin{array}{l}\text { Valor de la educación ( } 1=e s \text { la educación en la familia un valor importante, } 0 \text { = no es la } \\
\text { educación en la familia un valor importante) }\end{array}$ & 0,06 \\
\hline
\end{tabular}

Continúa... 
URL: http://www.una.ac.cr/educare

CORREO: educare@una.cr

\section{Variables}

Vive con los padres biológicos $(1=s i ́, 0=n o)$

Experimenta situaciones de violencia ( $1=$ experimenta violencia familiar actualmente, $0=$ no experimenta violencia familiar actualmente)

Experimentó situaciones de violencia (1= experimentó violencia familiar en el pasado, $0=$ no experimentó violencia familiar en el pasado)

Conversaciones de temas académicos en la familia(1= se da con frecuencia en el hogar conversaciones académicos, $0=$ no se da con frecuencia conversaciones académicas)

Convivencia familiar ( $1=$ considera que la convivencia familiar es regular y mala, $0=$ considera que la convivencia familiar es muy buena)

Relación con el padre (1= relación impositiva y de temor, $0=$ relación abierta al diálogo y de confianza)

Relación con la madre $(1=$ relación impositiva y de temor, $0=$ relación abierta al diálogo y de confianza)

Notas: *Es un valor constante no se puede calcular la correlación (no hay variabilidad, toda esta población reportó una buena relación con la madre). Correlación va de 0 a 1, cuanto más cercano a 1, mayor es la correlación o asociación con el rendimiento académico.

\section{Variables numéricas}

Las correlaciones de las variables numéricas se calcularon con la correlación de Pearson. Las variables continuas que presentan mayor asociación con el rendimiento académico (promedio ponderado) son:

- Escala de satisfacción de la carrera universitaria (Pearson $=0.205$, significancia $=0.119$ )

- Escala de apoyo a los estudios universitarios (Pearson $=0.310$, significancia $=0.017$ )

- $\quad$ Nota de admisión $($ Pearson $=0.383$, significancia $=0.003$ )

La nota de admisión es la que tiene la mayor asociación con el rendimiento académico. Este comportamiento se visualiza en la figura 1: 
URL: http://www.una.ac.cr/educare

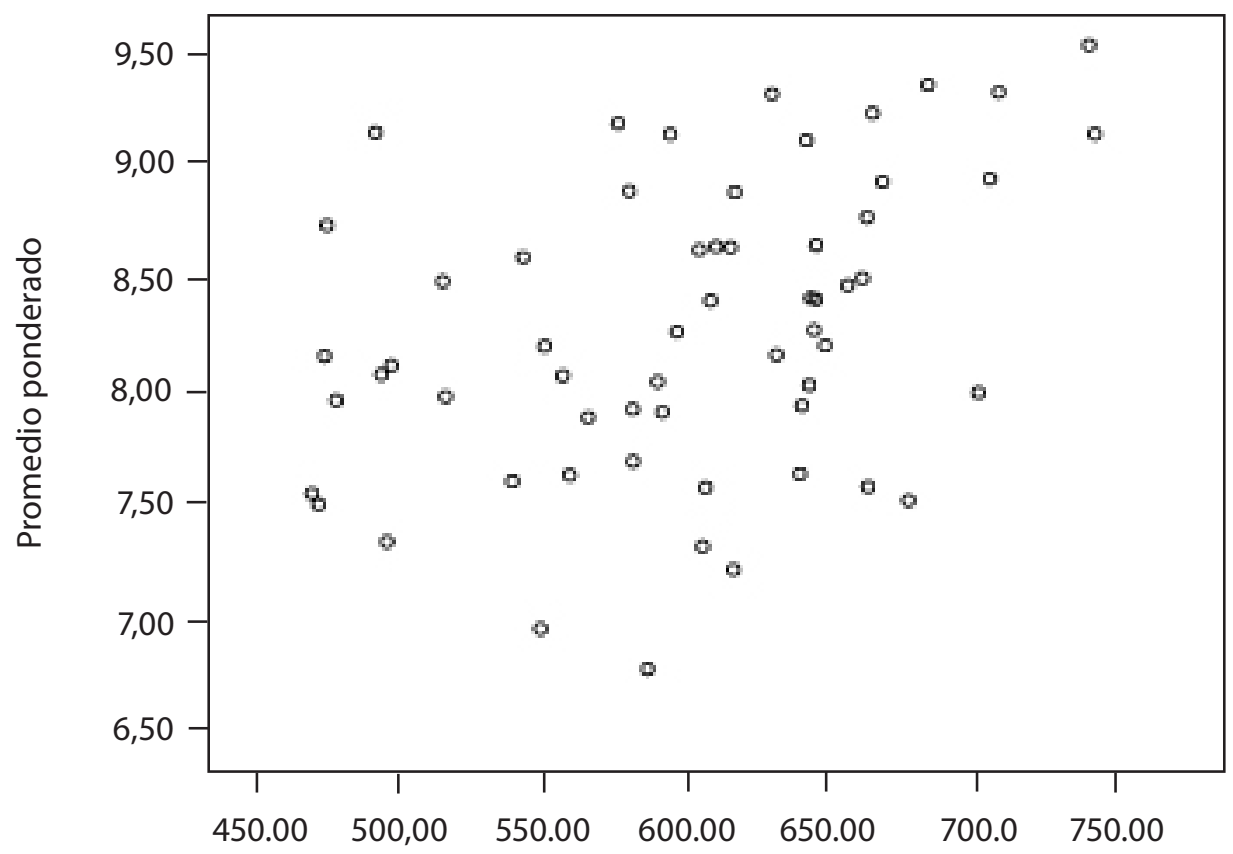

Nota de admisión

Figura 1. Nota de admisión y promedio ponderado población de alta demanda. Cuestionario "factores asociados al rendimiento académico" aplicado a estudiantes universitarios carreras de alta y baja demanda según condición socioeconómicos, es decir, para estos efectos estudiantes con la máxima categoría de beca por su condición socioeconómica y estudiantes que no solicitaron beca por esta condición.

Se realizó una prueba de medias, que mide si existe diferencias significativas en algunas variables como nota de admisión, promedio ponderado y las diferentes escalas, entre estudiantes con la máxima categoría de beca (beca 11) y estudiantes que no solicitaron beca (sin beca). Efectivamente, existen diferencias significativas en las siguientes variables: escala de satisfacción de la carrera universitaria, escala de factores intrínsecos académicos, escala de satisfacción con los profesores e índice de nivel socioeconómico; sin embargo, se mostró que esto no produce diferencias en las variables del promedio ponderado y la nota de admisión.

Sí existen diferencias entre algunas variables de la población de estudiantes que no solicitaron beca (sin beca) y con la población de estudiantes con la máxima categoría de beca (beca 11). El la tabla 5 se ilustra esta información: 
URL: http://www.una.ac.cr/educare

CORREO: educare@una.cr

Tabla 5

Prueba de medias para estudiantes sin beca y con beca 11 que estudian en carreras de alta demanda

\begin{tabular}{|c|c|c|c|c|c|}
\hline \multirow[b]{2}{*}{ Variable } & \multirow[t]{2}{*}{ Valor T } & \multirow[t]{2}{*}{ Significancia } & \multicolumn{2}{|c|}{ Promedios } & \multirow[t]{2}{*}{ Diferencia } \\
\hline & & & Sin beca & Beca 11 & \\
\hline Nota de admisión & 1,61 & 0,11 & 612,63 & 584,33 & 28,30 \\
\hline Promedio ponderado & 1,20 & 0,23 & 8,32 & 8,11 & 0,21 \\
\hline $\begin{array}{l}\text { Escala de satisfacción con la } \\
\text { carrera universitaria* }\end{array}$ & $-2,43$ & 0,02 & 15,36 & 16,79 & $-1,44$ \\
\hline $\begin{array}{l}\text { Escala de factores intrínsecos } \\
\text { académicos* }\end{array}$ & $-2,99$ & 0,00 & 26,47 & 28,38 & $-1,91$ \\
\hline Escala de hábitos de estudio & $-0,20$ & 0,84 & 8,48 & 8,59 & $-0,10$ \\
\hline $\begin{array}{l}\text { Escala de apoyo en los estudios } \\
\text { universitarios }\end{array}$ & 1,70 & 0,09 & 15,00 & 13,38 & 1,62 \\
\hline $\begin{array}{l}\text { Escala de satisfacción con los } \\
\text { profesores universitarios* }\end{array}$ & $-2,23$ & 0,03 & 29,60 & 31,86 & $-2,25$ \\
\hline $\begin{array}{l}\text { Escala de satisfacción con los } \\
\text { servicios }\end{array}$ & $-1,75$ & 0,09 & 9,13 & 9,98 & $-0,85$ \\
\hline Nivel socioeconómico* & 7,37 & 0,00 & 70,43 & 21,26 & 49,17 \\
\hline
\end{tabular}

Nota: *Significativamente diferentes a un nivel de significancia del 5\%.

\section{Correlación de variables independientes}

La correlación entre las variables independientes permite visualizar cuáles variables están siendo redundantes; ofrece un criterio para sacar algunas con estas características del modelo de regresión para que el modelo sea más eficaz y eficiente. Así, se eliminaron las variables que ofrecían la misma información. El criterio para la eliminación de las variables siguió los siguientes pasos:

1. Evaluar la correlación entre dos variables independientes, si es alta $(\rho>=0.70)$ y significativa alguna de las dos está elegible para eliminarla.

2. Se evalúa la correlación de cada una de ellas con el rendimiento académico, entonces la que tiene mayor correlación con el rendimiento es la que aporta más al modelo; por lo tanto, es la variable que se queda en el modelo. 
Sustentado en lo anterior, se realiza el modelo de regresión que se presenta en la tabla 6, en el cual no se incluyen todas las variables que se mencionaron. Se eliminaron las siguientes: cursó educación preescolar, modalidad de colegio, experimenta actualmente violencia familiar, cómo valora la relación con su padre, cómo valora la relación con su madre, vive con sus padres biológicos, nivel educativo del padre y valor de la educación.

Los coeficientes estimados por este modelo son:

Tabla 6

Coeficientes de regresión

\begin{tabular}{|c|c|c|c|c|c|c|c|c|c|}
\hline \multirow{2}{*}{ Modelo } & \multicolumn{2}{|c|}{$\begin{array}{l}\text { Coeficientes no } \\
\text { estandarizados }\end{array}$} & \multicolumn{3}{|c|}{$\begin{array}{l}\text { Coeficientes } \\
\text { estandarizados }\end{array}$} & \multicolumn{2}{|c|}{$\begin{array}{l}\text { Intervalos de } \\
\text { confianza }\end{array}$} & \multirow[t]{2}{*}{ FIV } & \\
\hline & B & Error tip. & Beta & $\mathrm{t}$ & Sig. & Inferior & Superior & & \\
\hline Constante & $-0,88$ & 2,35 & & $-0,38$ & 0,71 & $-5,63$ & 3,87 & & \\
\hline Sexo & $-0,27$ & 0,16 & $-0,20$ & $-1,71$ & 0,10 & $-0,58$ & 0,05 & 1,37 & * \\
\hline Edad & 0,08 & 0,06 & 0,16 & 1,26 & 0,21 & $-0,05$ & 0,21 & 1,61 & * \\
\hline Beca & $-0,62$ & 0,25 & $-0,47$ & $-2,47$ & 0,02 & $-1,13$ & $-0,11$ & 3,71 & $* *$ \\
\hline $\begin{array}{l}\text { Es la carrera que } \\
\text { deseaba estudiar }\end{array}$ & 0,53 & 0,19 & 0,31 & 2,82 & 0,01 & 0,15 & 0,91 & 1,30 & $* *$ \\
\hline $\begin{array}{l}\text { Escala de satisfacción } \\
\text { de la carrera } \\
\text { universitaria }\end{array}$ & 0,02 & 0,05 & 0,07 & 0,38 & 0,71 & $-0,08$ & 0,11 & 3,10 & \\
\hline $\begin{array}{l}\text { Escala de factores } \\
\text { intrínsecos }\end{array}$ & 0,08 & 0,04 & 0,33 & 2,17 & 0,04 & 0,01 & 0,16 & 2,42 & $* *$ \\
\hline $\begin{array}{l}\text { Escala de hábitos de } \\
\text { estudio }\end{array}$ & 0,04 & 0,01 & 0,11 & 0,96 & 0,34 & $-0,04$ & 0,12 & 1,45 & $*$ \\
\hline $\begin{array}{l}\text { Residencia en tiempo } \\
\text { lectivo }\end{array}$ & $-0,22$ & 0,19 & $-0,15$ & $-1,18$ & 0,25 & $-0,60$ & 0,16 & 1,63 & * \\
\hline Escala de apoyo & 0,03 & 0,02 & 0,19 & 1,27 & 0,21 & $-0,02$ & 0,09 & 2,26 & * \\
\hline $\begin{array}{l}\text { Nivel académico de la } \\
\text { madre }\end{array}$ & $-0,07$ & 0,07 & $-0,17$ & $-0,92$ & 0,36 & $-0,21$ & 0,08 & 3,48 & * \\
\hline $\begin{array}{l}\text { Conversaciones temas } \\
\text { académicos }\end{array}$ & 0,33 & 0,15 & 0,24 & 2,19 & 0,04 & 0,02 & 0,63 & 1,27 & $* *$ \\
\hline Convivencia familiar & 0,05 & 0,28 & 0,03 & 0,17 & 0,87 & $-0,52$ & 0,61 & 2,49 & \\
\hline
\end{tabular}

Continúa... 
URL: http://www.una.ac.cr/educare

CORREO: educare@una.cr

\begin{tabular}{|c|c|c|c|c|c|c|c|c|c|}
\hline \multirow[t]{2}{*}{ Modelo } & \multicolumn{2}{|c|}{$\begin{array}{l}\text { Coeficientes no } \\
\text { estandarizados }\end{array}$} & \multicolumn{3}{|c|}{$\begin{array}{l}\text { Coeficientes } \\
\text { estandarizados }\end{array}$} & \multicolumn{2}{|c|}{$\begin{array}{l}\text { Intervalos de } \\
\text { confianza }\end{array}$} & \multirow[t]{2}{*}{ FIV } & \\
\hline & B & Error tip. & Beta & $\mathrm{t}$ & Sig. & Inferior & Superior & & \\
\hline $\begin{array}{l}\text { Violencia familiar } \\
\text { pasada }\end{array}$ & $-0,03$ & 0,29 & $-0,02$ & $-0,10$ & 0,92 & $-0,62$ & 0,56 & 2,95 & \\
\hline Horas de trabajo & $-0,01$ & 0,01 & $-0,26$ & $-2,15$ & 0,04 & $-0,02$ & $-0,00$ & 1,55 & ** \\
\hline $\begin{array}{l}\text { Escala de satisfacción } \\
\text { con los profesores }\end{array}$ & 0,03 & 0,02 & 0,18 & 1,24 & 0,22 & $-0,02$ & 0,08 & 2,17 & * \\
\hline $\begin{array}{l}\text { Escala de satisfacción } \\
\text { con los servicios }\end{array}$ & 0,04 & 0,05 & 0,10 & 0,71 & 0,48 & $-0,07$ & 0,14 & 2,09 & * \\
\hline $\begin{array}{l}\text { Índice nivel } \\
\text { socioeconómico }\end{array}$ & 0,00 & 0,00 & 0,04 & 0,24 & 0,81 & $-0,01$ & 0,01 & 3,28 & \\
\hline Nota de admisión & 0,01 & 0,00 & 0,49 & 3,64 & 0,00 & 0,00 & 0,01 & 1,91 & ** \\
\hline
\end{tabular}

Notas: *Un asterisco indica que la variable independiente presenta una y solo una de las dos condiciones: un valor de beta igual o mayor que 0.10 (a partir del cual se considera relevante para la predicción a nivel descriptivo) o un valor de p menor a 0.05 indicando que el coeficiente asociado a la respectiva variable dependiente es estadísticamente significativo (es decir, se puede generalizar a toda la población). ${ }^{* *}$ Dos asteriscos indican que la variable independiente presenta las dos condiciones: un valor de beta igual o mayor a 0.10 (a partir del cual se considera relevante para la predicción a nivel descriptivo) y un valor de $p$ menor a 0.05 indicador de que el coeficiente asociado a la respectiva variable dependiente es estadísticamente significativo (es decir, se puede generalizar a toda la población). Esta anotación se tomó de Montero y Villalobos (2004b).

Este modelo presenta un $\mathrm{R}^{2}$ de 0.647 , con un error de estimación de 0.487 . La medida de $\mathrm{R}^{2}$ se denomina coeficiente de determinación y se utiliza para evaluar la capacidad predictiva del modelo de regresión. Su valor $\left(R^{2}\right)$ indica el porcentaje de variabilidad en la variable dependiente, lo cual podría ser explicado desde su relación lineal con las variables independientes.

Las siguientes variables resultaron ser significativas a nivel estadístico y, además, de importancia práctica, ya que $p<0.05$ y el valor de beta es mayor a 0.10 . Estas variables son las que presentan evidencia más fuerte de asociación con el rendimiento académico.

- Beca (condición socioeconómica). $B=-0.62$ (relación inversa)

- Es la carrera que deseaba estudiar. $B=0.53$ (relación directa)

- Escala de factores intrínsecos; motivación intrínseca. $B=0.08$ (relación directa)

- Conversaciones de temas académicos frecuentes en la familia. $B=0,33$ (relación directa)

- Horas de trabajo. $B=-0.01$ (relación inversa)

- Nota de admisión. $B=0.05$ (relación directa) 
Las siguientes variables resultaron de importancia práctica; sin embargo, no son significativas; es decir, el resultado que dio no se puede generalizar a nivel de toda la población; no obstante, son variables importantes para predecir el rendimiento académico: sexo, edad, escala de hábitos de estudio, residencia en tiempo lectivo, escala de apoyo, nivel académico de la madre, escala de satisfacción con los profesores y escala de satisfacción con los servicios.

- Variable "beca" (Variable dummy. 1=asignación de beca 11, 0= sin beca). La variable "beca", en este caso, la condición socioeconómica, es un factor asociado al rendimiento académico de índole social. Es una variable significativa, con un valor de 0,02 lo que permite generalizarla a la población. En este caso, es de importancia práctica, con un valor de beta de $-0,47$, a partir del cual se considera relevante para la predicción a nivel descriptivo. Posee una relación, estadísticamente significativa, con la variable dependiente (promedio ponderado).

En el caso de esta variable beca, las diferencias se prestan a favor de los estudiantes que no solicitaron beca, el coeficiente bruto de regresión (no estandarizado) es de -0,62; esto es una relación inversa, lo cual indica que, en promedio, y manteniendo constantes o controladas todas las otras variables independientes (Ceteris Paribus) en el modelo, cuando en los estudiantes de beca 11; es decir, con la máxima categoría de beca por su condición socioeconómica, el promedio ponderado baja en 0,62 puntos en promedio del rendimiento académico, en comparación con estudiantes sin beca; es decir, estudiantes que no solicitaron beca por condición socioeconómica.

Este comportamiento confirma lo que ya otros estudios han evidenciado en relación con los resultados académicos, donde la influencia del contexto socioeconómico está significativamente asociada con el rendimiento académico; en este caso, en estudiantes con la máxima categoría de beca su promedio ponderado disminuye en ,620 puntos promedio del promedio ponderado, en comparación con los estudiantes de que no solicitaron beca (Cohen, 2002). Factores como la pobreza y la falta de apoyo social se asocian con bajos resultados académicos (Marchesi, 2000).

- Variable "es la carrera que deseaba estudiar" (Variable dummy. 1=sí, 0= no). Es una variable de importancia práctica para predecir el rendimiento académico; posee un valor de Beta de 0,31 y es significativa con un valor de 0,01, lo que permite generalizarla a la población. En el caso de esta variable "es la carrera que deseaba estudiar" posee un coeficiente bruto de regresión (no estandarizado) de 0,53 , lo cual indica que manteniendo controladas o constantes todas las otras variables independientes (Ceteris Paribus) en el modelo, cuando los estudiantes ingresan en las carreras que querían estudiar, el promedio ponderado aumenta en 0,53 puntos en promedio del promedio ponderado, más que los que están en una carrera que no es la que querían estudiar. Es una variable de importancia práctica y significativa en la predicción del rendimiento académico.

Este hallazgo confirma la relación que se da entre el rendimiento académico y la satisfacción vocacional, con la selección de la carrera universitaria; estudiantes que cursan la carrera deseada presentan importantes motivaciones intrínsecas hacia la tarea académica; también se relaciona 
URL: http://www.una.ac.cr/educare

CORREO: educare@una.cr

con un alto autoconcepto académico y se produce un aumento en el promedio ponderado, en comparación con aquellos estudiantes que están cursando una carrera que no es la que deseaban estudiar (De Miguel et al., 2002).

- Variable "Escala de factores intrínsecos; motivación intrínseca". Esta escala está conformada por 10 ítems (escala 10-30). La escala valora factores motivacionales intrínsecos académicos del estudiante, relacionados con sus actitudes ante la vida académica, según los siguientes aspectos:

- Certeza de lo que se debe hacer para lograr las metas académicas.

- Esfuerzo personal en concordancia con resultados académicos.

- Capacidad de enfrentar los obstáculos que afectan la academia con esfuerzo.

- Alcanzar el éxito académico es una meta constante.

- Estudiar representa un disfrute.

- Entusiasmo para los estudios.

- Orgullo ante las actividades académicas.

- Estudios representan un reto a disfrutar.

- No se percibe que el tiempo pasa cuando es dedicado a las actividades académicas.

- Resultados académicos consecuentes con el desempeño académico.

La "escala de factores intrínsecos; motivación intrínseca" es un factor de índole personal (psicosocial), según la teoría, para predecir el rendimiento académico. Esta escala está conformada por una serie de actitudes del estudiante relacionadas con la motivación intrínseca ante el desempeño académico; en este estudio, es una variable de importancia práctica. Es importante para predecir el rendimiento académico; posee un valor de Beta de 0,33 y es significativa con un valor de 0,04, lo cual permite generalizarla a la población. Esta variable posee un coeficiente bruto de regresión (no estandarizado) de 0,08 , lo que indica que manteniendo controladas o constantes todas las otras variables independientes (Ceteris Paribus) en el modelo, cuando los estudiantes tienen alta su escala de factores intrínsecos, hay un aumento del promedio ponderado en 0,08 puntos en promedio del promedio ponderado más que aquellos estudiantes que poseen baja esta escala de motivación intrínseca.

Ese aumento significa que aquellos estudiantes que tuvieron una motivación intrínseca positiva para enfrentar las demandas del desempeño académico tienden a tener una mayor capacidad académica, por lo cual este positivismo se ve reflejado en un aumento en el promedio ponderado.

Lo anterior viene a confirmar lo expuesto en este campo, en el que se dice que aquellos estudiantes para quienes el estudio representa un alto nivel de motivación intrínseca académica 
(el estudio significa un disfrute, no perciben que el tiempo pasa cuando están dedicados a sus estudios, presentan una buena disposición hacia la tarea académica, experimentan sentimientos de felicidad hacia la vida académica, se proponen objetivos a alcanzar académicamente, no se rinden ante los obstáculos académicos, tienen el éxito académico como una meta constante, como un reto que se disfruta) son estudiantes que, debido a estas herramientas motivadoras, tienen persistencia y logran buenos resultados en el desempeño académico (Salanova, Cifre, Grau, Llorens, y Martínez. (2005).

- Variable "conversaciones frecuentes sobre temas académicos en la familia" (dummy $1=$ se dan con frecuencia en el hogar conversaciones académicos, $0=$ no se dan con frecuencia conversaciones académicas). Esta es una variable de importancia práctica, al respecto, reviste relevancia para predecir el rendimiento académico, posee un valor de Beta de 0,24 y es significativa con un valor de 0,04, lo cual permite generalizarla a la población.

Esta variable "conversaciones sobre temas académicos en la familia" se refiere a estudiantes que en sus hogares tienen conversaciones constantes sobre temas académicos, poseen un coeficiente bruto de regresión (no estandarizado) de 0,33, lo que indica que manteniendo controladas o constantes todas las otras variables independientes (Ceteris Paribus) en el modelo, cuando los estudiantes tienen en sus hogares frecuentes conversaciones sobre temas académicos, aumenta del promedio ponderado en 0,33 puntos en promedio del promedio ponderado más de aquellos estudiantes cuyos hogares no se caracterizan por la frecuencia de las conversaciones sobre temas académicos. Es una variable predictiva del rendimiento académico de tipo social.

Se podría inferir que, cuando hay un buen entorno familiar donde los temas académicos son importantes, se estimula en los estudiantes mayor compromiso con el estudio; lo cual se evidencia con el aumento en el promedio ponderado de 0,33 puntos en promedio.

Al analizar las diferencias entre estudiantes que no solicitaron beca (sin beca) y los estudiantes con la máxima categoría de beca (con beca), son los estudiantes becados 11 (estudiantes con la máxima categoría de beca por su condición socioeconómica) los que en sus hogares poseen con menos frecuencia las conversaciones académicas; sin embargo, esta diferencia no resultó significativa en esta población.

También, esta variable presentó una baja asociación con el promedio ponderado $(0,14)$. Al respecto, Kellaghan y Álvarez, citados por Gómez (1998) coinciden en que el rendimiento académico de los estudiantes se encuentra ligado al ambiente de aprendizaje que la familia proporciona. Ambientes familiares donde se estimula el placer por los estudios, la perseverancia hacia el logro académico y la inquietud por el saber, se asocian con buenos resultados académicos (Pelegrina, García y Casanova, 2002).

- Variable "horas de trabajo" (horas de trabajo por semana, $0=$ no trabaja). En cuanto a la variable "horas de trabajo", es una variable significativa con un valor de 0,04 , lo que permite generalizarla a la población, posee importancia práctica, con un valor de Beta de -0,26. 
En el caso de los estudiantes que laboran, se encontró una relación estadísticamente significativa con la variable dependiente. En los estudiantes con mayor cantidad de horas de trabajo, disminuye el promedio en 0,01 puntos, en promedio, del promedio ponderado; se da un coeficiente bruto de regresión (no estandarizado) de - -0,01, lo cual indica que manteniendo controladas o constantes todas las otras variables independientes (Ceteris Paribus) en el modelo, su promedio disminuye en 0,01 puntos en promedio del promedio ponderado que los estudiantes que no trabajan. Se puede deducir que esta variable tiene relación con la disposición de tiempo de los estudiantes con las obligaciones académicas.

Las horas que dedica un estudiante universitario para laborar podrían tener relación con su contexto socioeconómico. Al respecto, Cohen (2002) llama la atención sobre la relación de este contexto y resultados académicos. Sin embargo, para este caso, sería incorrecto afirmar o pensar que las personas que laboran más horas son de una condición socioeconómica baja, puesto que en este grupo prácticamente todos los que laboran son estudiantes de Derecho, que no solicitaron beca, lo que podría deberse a las características de esta carrera, donde el adquirir experiencia laboral es fundamental; se insertan rápidamente en la vida laboral, razón por la cual esta situación podría verse como una excepción.

- Variable "nota de admisión" (puntaje obtenido en el promedio de admisión para ingresar a la universidad, está compuesto de un 50\% del examen de admisión (PPA), y un 50\% de las notas de los últimos 5 trimestres del colegio (escala de 400-800). La nota de admisión, denominada en la Universidad de Costa Rica como Prueba de Aptitud Académica (PAA), es un instrumento psicométrico de alta calidad técnica, donde se combinan con la misma ponderación la nota del examen de admisión y el promedio de las materias básicas de educación diversificada. Es un indicador de destrezas o habilidades académicas intelectuales. Mediante la nota de admisión, se pretende seleccionar a aquellos estudiantes que tienen mayores probabilidades de éxito en la vida universitaria dentro de una carrera (Montero y Villalobos, 2004b).

La "nota de admisión" también resultó estadísticamente significativa y de importancia práctica para predecir el promedio ponderado del estudiante. De hecho, presenta el coeficiente Beta más elevado en el grupo de las variables independientes (Beta=0,49). Es significativa con un valor de 0,00, lo cual permite generalizarla a la población.

Lo anterior viene a confirmar que la "nota de admisión" es un instrumento adecuado para la selección de estudiantes en el ingreso a la universidad y un predictor del rendimiento académico de importancia. Sin embargo, el cambio ocasionado por esta en el promedio ponderado es relativamente bajo, $B=0.01$. Lo anterior podría obedecer a que en el grupo de las carreras de alta demanda, por sus características competitivas de oferta y demanda, sus estudiantes ingresan, por lo general, con promedios de admisión altos; son estudiantes académicamente con un buen desempeño; las diferencias en los promedios de admisión entre estudiantes con la máxima categoría de beca o estudiantes que no solicitaron beca no son importantes, razón por la cual no hay diferencias significativas en su rendimiento académico, sus promedios no son estadísticamente diferentes. 
El estudio permite, una vez más, demostrar el valor predictivo que tiene la nota de admisión en el rendimiento académico del estudiante; Montero y Villalobos (2004b) coinciden en que es uno de los predictores más importantes en el rendimiento académico del estudiante universitario; Toca y Tourón (1989) también coinciden en este valor predictivo.

Las siguientes variables resultaron de importancia práctica; sin embargo, no son significativas; es decir, el resultado que dio no se puede generalizar estadísticamente a nivel de toda la población; no obstante, estas variables sí tienen valor predictivo para predecir el rendimiento académico para los datos de la muestra: sexo del estudiante, la edad, escala de hábitos de estudio, residencia en tiempo lectivo, escala de apoyo en los estudios, nivel académico de la madre, escala de satisfacción con los profesores y escala de satisfacción con los servicios universitarios.

Índice de nivel socioeconómico: sí existen diferencias en el nivel socioeconómico entre la gente con la máxima categoría de beca (con beca) y los estudiantes que no solicitaron beca (sin beca); sin embargo, se puede ver que el nivel socioeconómico valorado en este índice no es una variable que predice el promedio ponderado, según el modelo de regresión del estudio.

Escala de satisfacción con la carrera universitaria: sí existe diferencia con la satisfacción con la carrera universitaria que tienen los estudiantes con la máxima categoría de beca (becados 11) y los que no solicitaron beca (sin beca) 16.79 y 15.36 respectivamente; es decir, según su condición socioeconómica. Los estudiantes con la máxima categoría de beca tienen más alta su escala de satisfacción respecto de su carrera universitaria que quienes no solicitaron beca. Esta escala es un factor de importancia práctica para determinar el promedio ponderado, según el modelo de regresión del estudio.

Escala de factores intrínsecos académicos: sí existe diferencia con los factores intrínsecos académicos que tienen los estudiantes con la máxima categoría de beca (becados 11) y los que no solicitaron beca (sin beca). El grado de los factores intrínsecos es un factor que determina el promedio ponderado, resultó ser una variable significativa a nivel estadístico y de importancia práctica para la predicción del rendimiento académico, según el modelo de regresión del estudio.

Dada la importancia de esta variable para predecir el rendimiento académico en esta población de alta demanda, es importante retomar el hecho de que los estudiantes con la máxima categoría de beca (becados 11) son los que evidencian la diferencia más alta en la escala de factores intrínsecos $(28,38)$ que los que no solicitaron beca (sin beca) $(26,47)$, por lo cual podría inferirse que aquellos estudiantes con la máxima categoría de beca, para llegar donde han llegado académicamente, han desarrollado una fuerte motivación intrínseca para la tarea académica, debido, entre algunas razones, a las desventajas socioeconómicas que esta población trae consigo, las cuales podrían ser limitantes para la continuación de sus estudios universitarios. Esta situación es respaldada teóricamente por autores como Rutter (1993), citado por Badilla y Sancho (1997), al referirse a la capacidad de algunas personas de triunfar a pesar de las adversidades de sus ambientes; expuestos en la teoría de la resiliencia. Esta teoría hace referencia a la capacidad de reaccionar positivamente, a pesar de las dificultades que el individuo enfrenta; situación que parece 
URL: http://www.una.ac.cr/educare

CORREO: educare@una.cr

estar latente en este estudio con los estudiantes con la máxima categoría de beca por su condición socioeconómica.

Escala de hábitos de estudio: sí existe diferencia con los hábitos de estudio que tienen los estudiantes con la máxima categoría de beca (becados 11) y los que no solicitaron beca (sin beca); sin embargo, el grado de los hábitos de estudio no es un factor de significancia en la predicción del promedio ponderado, aunque sí posee importancia práctica, según el modelo de regresión del estudio.

Escala de apoyo en los estudios universitarios: con sustento en la prueba de medias, se encontró que sí hay diferencias significativas en la escala de apoyo en los estudios universitarios de los estudiantes con la máxima categoría de beca (con beca 11); y estudiantes que no solicitaron beca (sin beca): (13.38 y 15.00 respectivamente), es decir, según su condición socioeconómica. Así las cosas, los estudiantes becados 11 tienen más apoyo para sus estudios que los que no tienen beca. El grado de apoyo a los estudios universitarios no es un factor de significancia en la predicción del promedio ponderado, aunque sí posee importancia práctica, según el modelo de regresión del estudio.

Respecto de las variables categóricas (tabla 3) se encontraron diferencias significativas en la población con la máxima categoría de beca y los estudiantes que no solicitaron beca en la población de alta demanda con la variable "modalidad de colegio" y "asistencia a educación preescolar".

En la modalidad de colegio, hay diferencias significativas en ambos grupos. La mayoría de estudiantes con la máxima categoría de beca (becados) en el grupo de alta demanda proviene de colegios públicos; lo mismo sucede en el grupo de baja demanda.

Respecto de la asistencia a educación preescolar, los estudiantes con la máxima categoría de beca son los que mayormente indican no haber asistido a educación preescolar, mientras los estudiantes que no solicitaron beca (sin beca), en su mayoría, sí asistieron a educación preescolar. Sin embargo, ninguna de estas variables resultó ser de importancia práctica y significativa para predecir el rendimiento académico.

En el presente estudio, las variables independientes que resultaron con valores de beta de importancia práctica mayores a 0,10 y también significativas a nivel estadístico, que presentan una fuerte asociación con el rendimiento académico, en la población de estudiantes de carreras de alta demanda, son las siguientes:

- Beca: asignación de beca 11 y estudiantes sin beca; estudiantes con la máxima categoría de beca por su condición socioeconómica y estudiantes que no solicitaron beca por condición socioeconómica (relación inversa).

- Es la carrera que desea estudiar (relación directa).

- Escala de factores intrínsecos (relación directa).

- Conversaciones de temas académicos en el hogar (relación directa). 
URL: http://www.una.ac.cr/educare CORREO: educare@una.cr

- Horas de trabajo (relación inversa).

- La nota de admisión (relación directa).

Estos factores se deben considerar de manera especial, pues su presencia predice de manera importante del rendimiento académico en este grupo, de manera que son acreedores de significancia e importancia práctica.

Las siguientes variables resultaron ser de importancia práctica; sin embargo, no son significativas para predecir el rendimiento académico:

- Sexo del estudiante (relación inversa)

- La edad (relación directa)

- Escala de hábitos de estudio (relación directa)

- Residencia en tiempo lectivo (relación inversa)

- Escala de apoyo en los estudios (relación directa)

- Nivel académico de la madre (relación inversa)

- Escala de satisfacción con los profesores (relación directa)

- Escala de satisfacción con los servicios universitarios (relación directa)

\section{Grupo de estudiantes de carreras de baja demanda: \\ Diferencias de medias}

\section{Variables numéricas}

Las únicas variables que en ambos grupos, es decir, en las carreras de alta y baja demanda, presentan diferencias significativas entre estudiantes con la máxima categoría de beca (beca 11) y los que no solicitaron beca (sin beca), es la escala de apoyo a los estudios universitarios y, por supuesto, el nivel socioeconómico; sin embargo, estas variables no resultaron de importancia práctica y significativa en la predicción del rendimiento académico.

\section{Variables categóricas}

Al igual que en el grupo de estudiantes de carreras de alta demanda, se calculó el estadístico Eta, para medir la asociación entre cada una de las variables categóricas del estudio y el promedio ponderado.

Lo curioso es que en este grupo, prácticamente ninguna de estas variables está mediana o altamente correlacionada con el rendimiento académico. De hecho, la variable con mayor correlación es la violencia familiar actual y, aun así, la correlación es baja (0.17). 
URL: http://www.una.ac.cr/educare

CORREO: educare@una.cr

\section{Correlación de variables independientes}

El primer modelo de regresión incluyó todas las variables contempladas en el estudio; el modelo no resulta significativo y tiene problemas de multicolinealidad; por lo tanto, se eliminan algunas de las variables con mayor correlación con el resto de las predictivas y con menor correlación con el promedio.

Las variables eliminadas son: nivel educativo de la madre, nivel educativo del padre, horas de trabajo, escala de hábitos de estudio, conversaciones de temas académicos, lugar de residencia en tiempo lectivo, cursó educación preescolar, relación con la madre, cómo considera la convivencia familiar, y modalidad de colegio y nivel socioeconómico.

Con estas variables eliminadas, el modelo es el siguiente (ver tabla 7):

Tabla 7

Coeficientes de regresión: baja demanda estudiantes con beca o sin beca

\begin{tabular}{|c|c|c|c|c|c|c|c|c|}
\hline \multirow{2}{*}{ Modelo } & \multicolumn{2}{|c|}{$\begin{array}{l}\text { Coeficientes no } \\
\text { estandarizados }\end{array}$} & \multicolumn{3}{|c|}{$\begin{array}{l}\text { Coeficientes } \\
\text { estandarizados }\end{array}$} & \multicolumn{2}{|c|}{$\begin{array}{l}\text { Intervalos de } \\
\text { confianza }\end{array}$} & \multirow[t]{2}{*}{ FIV } \\
\hline & B & $\begin{array}{l}\text { Error } \\
\text { tip. }\end{array}$ & Beta & $\mathrm{T}$ & Sig. & Inferior & Superior & \\
\hline Constante & 4,523 & 4,183 & & 1,081 & 0,292 & $-7,413$ & 20,131 & \\
\hline Sexo & $-0,05$ & 0,434 & $-0,029$ & $-0,116$ & 0,909 & $-2,501$ & 0,971 & 2,792 \\
\hline Edad & 0,065 & 0,097 & 0,151 & 0,664 & 0,514 & $-0,154$ & 0,364 & 1,652 \\
\hline Beca & $-0,21$ & 0,438 & $-0,125$ & $-0,48$ & 0,637 & $-1,915$ & 1,682 & 3,970 \\
\hline $\begin{array}{l}\text { Es la carrera que deseaba } \\
\text { estudiar }\end{array}$ & $-0,106$ & 0,454 & $-0,06$ & $-0,233$ & 0,818 & $-1,097$ & 1,39 & 1,690 \\
\hline $\begin{array}{l}\text { Escala de satisfacción } \\
\text { universitaria }\end{array}$ & 0,162 & 0,111 & 0,419 & 1,462 & 0,159 & $-0,081$ & 0,58 & 2,318 \\
\hline $\begin{array}{l}\text { Escala de factores intrínsecos } \\
\text { académicos }\end{array}$ & $-0,008$ & 0,075 & $-0,026$ & $-0,111$ & 0,913 & $-0,166$ & 0,293 & 1,908 \\
\hline $\begin{array}{l}\text { Escala de apoyo en los estudios } \\
\text { universitarios }\end{array}$ & 0,04 & 0,059 & 0,195 & 0,683 & 0,502 & $-0,156$ & 0,165 & 2,347 \\
\hline Valor de la educación & 0,428 & 0,821 & 0,146 & 0,521 & 0,608 & $-2,934$ & 2,851 & 4,523 \\
\hline $\begin{array}{l}\text { Experimentó situaciones de } \\
\text { violencia familiar }\end{array}$ & $-0,056$ & 0,889 & $-0,025$ & $-0,062$ & 0,952 & $-2,066$ & 1,955 & 2,776 \\
\hline $\begin{array}{l}\text { Escala de satisfacción con los } \\
\text { profesores }\end{array}$ & $-0,036$ & 0,057 & $-0,167$ & $-0,636$ & 0,532 & $-0,243$ & 0,076 & 1,742 \\
\hline $\begin{array}{l}\text { Escala de satisfacción con los } \\
\text { servicios }\end{array}$ & $-0,15$ & 0,195 & $-0,394$ & $-0,77$ & 0,461 & $-0,592$ & 0,291 & 4,448 \\
\hline Vive con sus padres biológicos & $-0,137$ & 0,758 & $-0,081$ & $-0,181$ & 0,860 & $-1,853$ & 1,578 & 3,410 \\
\hline Relación con el padre & 0,116 & 0,746 & 0,057 & 0,155 & 0,880 & $-1,572$ & 1,804 & 2,323 \\
\hline Nota de admisión & $-0,004$ & 0,006 & $-0,23$ & $-0,629$ & 0,545 & $-0,016$ & 0,009 & 2,276 \\
\hline
\end{tabular}


Este modelo resultó ser no significativo. Como en este análisis la prueba de hipótesis es que los coeficientes del modelo son iguales a 0 y no la significancia es $>0.05$, entonces no se rechaza esta hipótesis; es decir, este modelo de regresión no tiene en promedio coeficientes diferentes de 0 . Esto nos indica que el modelo con las variables predictivas escogidas no está prediciendo el rendimiento académico de manera precisa y confiable.

Se corrieron varios modelos, sin embargo, el único que resultó significativo incluye solamente dos variables predictivas: escala de satisfacción universitaria y escala de satisfacción con los servicios que brinda la universidad. No obstante, el porcentaje de variabilidad del rendimiento académico explicado por este modelo es bastante bajo $\left(\mathrm{R}^{2}=0.254\right)$ y, aún así, en este modelo solamente la satisfacción con la carrera universitaria resultó ser significativa, ambas sí son consideradas de relevancia para explicar el rendimiento académico.

Las correlaciones encontradas resultaron muy bajas; las variables que presentan una mayor asociación con el promedio ponderado en este grupo son:

- Escala de apoyo a los estudios universitarios

- Escala de satisfacción relacionado con la carrera universitaria

- Escala de factores intrínsecos académicos; motivación intrínseca

- Nivel académico del padre

Otro detalle por rescatar aquí es que la nota de admisión, que resultó ser una variable con una correlación importante con el rendimiento académico en el grupo de alta demanda, en este grupo fue una de las más bajas $(0,08)$; por lo tanto, resultó ser una correlación no significativa.

Respecto de las diferencias entre los estudiantes con beca 11 y sin beca, en la población de carreras de baja demanda, se puede decir que existen diferencias entre: el "lugar de residencia en época lectiva", la "modalidad de colegio", la presencia de "conversaciones académicas en el hogar" y si se ha "experimentado en el pasado situaciones de violencia familiar".

De las variables que resultaron con diferencias significativas en el grupo de baja demanda, ninguna de estas resultó ser significativa y de importancia práctica para predecir el rendimiento académico en el modelo. En el grupo de baja demanda, por lo menos en el modelo planteado con todas estas variables, no es el adecuado para predecir el rendimiento académico, lo cual indica que se debe buscar, profundizar e indagar en los factores que se asocian con el rendimiento específicamente en esta población. Hay algo más en ellos que hace que su promedio cambie, pero con este modelo no fue posible saberlo. Esta situación, dada en las carreras de baja demanda, permite reflexionar y generar algunas hipótesis al respecto: 
Sobre el sistema de ingreso a carrera que la universidad posee: se podría inferir que refleja la dinámica que desde hace mucho se da en esta institución, donde estudiantes que ven frustradas sus posibilidades de ingreso a carreras catalogadas como de "alta demanda" optan por asegurarse un espacio en carreras de baja demanda, sin que, vocacionalmente, implique una articulación con el estudiante en este sentido; y a su vez, estudiantes de buen promedio en la Prueba de Aptitud Académica ocupen un espacio en carreras de baja demanda, aunque no sea en la carrera de su agrado, con el único propósito de entrar a la universidad; espacio que otros estudiantes realmente necesitan según sus intereses vocacionales. Todo ello, producto de políticas institucionales que en cierta medida violentan el principio de excelencia académica.

El principio de excelencia académica también se podría lesionar en la medida en que estudiantes con altos promedios de admisión no encuentran la posibilidad de ingresar a la carrera deseada, y en lugar de optar por ingresar a carreras de baja demanda, como suele suceder, la decisión sea la de abandonar la universidad y colocarse en otras universidades; de esta manera, la Universidad de Costa Rica demuestra incapacidad para captar a los mejores estudiantes.

Finalmente, el comportamiento de esta población permite reflexionar sobre si el sistema de ingreso a carrera que posee la Universidad de Costa Rica es problemática; de ser así, sería posible que este sistema violente el principio de excelencia académica; estaría despreciando el talento, no necesariamente, solo los mejores estudiantes podrían estar ingresando a carrera, y estudiantes que ingresan de manera eventual, no necesariamente, por la excelencia terminen siendo un problema para la propia universidad.

\section{Conclusiones}

Tal y como se desprende del presente estudio, son muchas las interrogantes que deja expuestas el tema del rendimiento académico en la población estudiantil en la educación universitaria, en este caso específico en la Universidad de Costa Rica, donde se abordó la temática desde el nivel socioeconómico, tomando en cuenta el componente de la demanda de las distintas carreras universitarias.

La investigación permitió determinar, mediante modelos de regresión múltiple, una serie de factores psicosociales, institucionales, sociodemográficos y pedagógicos, asociados al rendimiento académico en estudiantes que acceden a carreras de alta demanda, dejando claro que hay diferencias importantes entre la población con la máxima categoría de beca por condición socioeconómica y aquellos que no solicitaron este tipo de beca; elementos que hasta ahora no se tenían en forma precisa. Sin embargo, deja sin respuesta los posibles factores que se asocian al rendimiento académico en aquellos estudiantes que acceden a carreras de baja demanda dentro de la Universidad de Costa Rica, debido a que el modelo de regresión utilizado no logró encontrar variables predictivas, como sí lo hizo con la población que accede a carreras de alta demanda. Esta situación induce a una reflexión y deja interrogantes, sobre la situación 
que viven los estudiantes que acceden a carreras de baja demanda, con el actual sistema de admisión a la universidad. Esta temática merece continuar investigándose, el cual puede ofrecer diferentes marcos explicativos dependiendo de la naturaleza de la disciplina en que se retome, así como su enfoque.

Al respecto, cobra especial interés el abordaje desde las clases sociales estudiantiles que la universidad atiende, la eficiencia del modelo de admisión imperante en esta casa de estudios en relación con la pertinencia social que como universidad pública le corresponde. No menos importante resulta el tema de la elección vocacional del estudiantado y las posibilidades que le ofrece la institución para acceder a la carrera deseada. Estas líneas de investigación quedan abiertas e institucionalmente es necesario retomarlas y continuar aportando al desarrollo de la universidad con respecto a su papel social, económico, político y cultural en la sociedad costarricense.

\section{Referencias}

Armenta, N. G., Pacheco, C. C. y Pineda, E. D. (2008). Factores económicos que intervienen en el desempeño académico de los estudiantes universitarios de la Facultad de Ciencias Humanas de la Universidad autónoma de Baja California. Revista IIPSI, 11(1), 153-165. Recuperado de http://sisbib.unmsm.edu.pe/bvrevistas/investigacion psicologia/v11 n1/pdf/a10.pdf

Badilla, E. y Sancho, M. (1997). Las experiencias de resiliencia como eje para un trabajo social alternativo (Tesis de licenciatura). Universidad de Costa Rica, San José, Costa Rica.

Cañedo, R. (2008). Educación superior y mercado de trabajo: El caso de los egresados de la Universidad Autónoma de Guerrero. (Tesis doctoral inédita). Universidad de Costa Rica, San José, Costa Rica.

CEPAL. (2000). Universidad y desarrollo. Proyecto latinoamericano. México: Autor.

Cohen, E. (setiembre-diciembre, 2002). Educación, eficiencia y equidad: Una difícil convivencia. Revista Iberoamericana de Educación, 30, 105-124. Recuperado de http://www.rieoei.org/ rie30a04.PDF

De Miguel, M., Apocada, P., Arias. J. M., Escudero, T., Rodríguez, S. y Vidal, J. (2002). Evaluación del rendimiento en la enseñanza superior. Comparación de resultados entre alumnos procedentes de la LOGSE y del COU. Revista de Investigación Educativa, 20(2), 357-383. Recuperado de http://revistas.um.es/rie/article/view/98971/94561

Freire, M. J. (abril, 2000). The Equality of Opportunities in the Access of Higher Education [La igualdad de oportunidades en el acceso a la educación superior]. Education Policy Análysis Archivies, 8(20), 1-17. Recuperado de http://epaa.asu.edu/ojs/article/view/411/534 
Gómez, H. (1998). Educación: La agenda del siglo XXI. Hacia un desarrollo humano. Bogotá: T/M Editores.

González, M. (2000). Niveles socioeconómicos AMAI. Recuperado de http://segmento.itam.mx/ Administrador/Uploader/material/Niveles Socioeconomicos AMAI.PDF

Jiménez, R., Robles, E. y Arce, G. (2009). Educación y crecimiento económico en Costa Rica. En L. Mesalles y O. Céspeces (Eds.), Obstáculos al crecimiento económico de Costa Rica (pp. 181-223). San José, Costa Rica: Academia de Centroamérica. Recuperado de http://www. academiaca.or.cr/documentos/Libroanual conportda 09.pdf

Jonson, R. (1988). Estadística elemental. México: Grupo Editorial Iberoamérica.

Larrañaga, O. (1997). Educación y superación de la pobreza en América Latina. Un estudio del proyecto "Mitigación de la pobreza y desarrollo social del programa de las Naciones Unidas para el Desarrollo. Suecia: PNUD. Recuperado de http://www.fuac.edu.co/download/ AREAS/2esx.pdf

Marchesi, Á. (mayo-agosto, 2000). Un sistema de indicadores de desigualdad educativa. Revista Iberoamericana de Educación, 23, 135-163. Recuperado de http://www.rieoei.org/rie23a04. PDF

Martínez, E. (2005). Errores frecuentes en la interpretación del coeficiente de la determinación del coeficiente lineal. Anuario Jurídico y Económico Escurialense, 37, 315-332. Recuperado de http://www.rcumariacristina.com/wp-content/uploads/2010/12/11-Elena-Martinez 1.pdf

Montero, E. (2008). Escalas o índices para la medición de constructos: El dilema del análisis de datos. Avances en Medición, 6(1), 15-24.

Montero, E. y Villalobos, J. (2004a). Estudio comparativo del promedio de admisión a la Universidad de Costa Rica y sus componentes: Aplicación del año 2001. San José, Costa Rica: SIEDIN.

Montero, E., Villalobos, J. (2004b). Factores institucionales, pedagógicos, psicosociales y sociodemográficos asociados al rendimiento académico y a la repetición estudiantil en la Universidad de Costa Rica. (Informe final proyecto 723-A0-174). San José, Costa Rica: Universidad de Costa Rica, Instituto de Investigaciones Psicológicas.

Montero, E., Villalobos, J. y Valverde, A. (2007). Factores institucionales, pedagógicos, psicosociales y sociodemográficos asociados al rendimiento académico en la Universidad de Costa Rica: Un análisis multinivel. RELIEVE, 13(2), 215-234. Recuperado de http://www.uv.es/RELIEVE/ v13n2/RELIEVEv13n2 5.pdf

Pelegrina, S., García, M. C. y Casanova, P. F. (2002). Los estilos educativos de los padres y la competencia académica de los adolescentes. Infancia y aprendizaje. Journal for the Study of Education and Development, 25(2), 147-168. 
Pérez, A., Ramón, J. y Sánchez, J. (2000). Análisis exploratorio de las variables que condicionan el rendimiento académico. Sevilla: Universidad Pablo de Olavide.

Pirrón, M., Rojas, M. y Arzola, V. M. (abril-junio, 2009). Planteamiento de la investigación sobre el nivel socioeconómico de los estudiantes que ingresan al nivel superior. Negotia Revista de Investigación de Negocios, 5(19), 23-33. Recuperado de http://www.sepi.escatep. ipn.mx/wps/wcm/connect/5F85A2804084333694C7DCEA05875C1/NEGOTIA193BCD. PDF?MOD=AJPERES

Plasencia, S. R., Vizconde, L. E., Ruiz, I. R., Araujo, M. E. y Salazar P. (2008). Estudio: Factores socioeconómicos y su relación con el rendimiento académico en estudiantes de la Universidad Nacional de Cajamarca. Recuperado de http://nuevo.unc.edu.pe/paginas/educacion/ IIEDUCA/Archivos/Articulo\%20Cientifico\%20-\%20PLASENCIA\%20CARRERA.pdf

Programa Estado de la Nación en Desarrollo Humano Sostenible. (2009). Decimoquinto Informe Estado de la Nación en Desarrollo Humano Sostenible. San José, Costa Rica: Autor.

Ramos, V. (2000). La educación y la circularidad de la pobreza: Resultados de la encueta nacional de hogares 1997-1998. Perú: Instituto Nacional de Estadística e Informática.

Rivero, H. (mayo-agosto, 1999). ¿Equidad en la educación? Revista lberoamericana de Educación, 23, 1-26. Recuperado de http://www.rieoei.org/rie23a03.htm

Rodríguez, S., Fita, E., Torrado, M. (mayo-agosto, 2004). El rendimiento académico en la transición secundaria-universidad. Revista de Educación, 334, 391-414. Recuperado de http://www. doredin.mec.es/documentos/008200430373.pdf

Ruh, P. (1998). Uso del bachillerato y del promedio de admisión como mecanismo para seleccionar a los estudiantes de colegio, que ingresan a la Universidad de Costa Rica. (Tesis de maestría inédita). Universidad de Costa Rica. San José, Costa Rica.

Ruiz, A. (2001). El destino de Costa Rica y la educación superior: El escenario histórico del país, la educación y el papel de la Universidad. San José, Costa Rica: EUCR.

Salanova, M., Cifre, E., Grau, R. M., Llorens, S. y Martínez, I. M. (2005). Antecedentes de la autoeficacia en profesores y estudiantes universitarios: Un modelo causal. Revista de Psicología del Trabajo y de las Organizaciones, 21(1-2), 159-176. Recuperado de http://www. redalyc.org/articulo.oa?id $=231317039010$

Toca, M. T. y Tourón, J. (1989). Factores del rendimiento académico en los estudios de arquitectura. Revista de Investigación Educativa, RIE, 7(14), 31-48.

UNESCO. (2002). Primera reunión intergubernamental del proyecto regional de educación para América Latina y el Caribe. Proyecto Regional de Educación para América Latina y El Caribe. Recuperado de http://www.tarea.org.pe/images/PRELAC Educ.pdf 


\section{URL: http://www.una.ac.cr/educare}

CORREO: educare@una.cr

Universidad de Costa Rica. Oficina de Becas y Atención Socioeconómica. (2009). Becas. San José, Costa Rica: Autor.

Universidad de Costa Rica., Oficina de Registro. (2003). Base de datos de la Oficina de Registro de la Universidad de Costa Rica. San José, Costa Rica: Autor.

Valverde, A. (2005). Factores asociados al rendimiento académico en la Universidad de Costa Rica: Un modelo estructural de variables latentes. (Tesis de licenciatura). Universidad de Costa Rica, San José, Costa Rica.

Vargas, M. M. (2010). Factores que determinan el rendimiento académico en matemáticas en la Universidad Nacional de Ingeniería, Nicaragua: Un estudio multinivel y de ecuaciones lineales estructurales (Tesis doctoral). Universidad de Costa Rica, San José, Costa Rica.

Vélez, A. y Roa, C. N. (2005). Factores asociados al rendimiento académico en estudiantes de medicina. Educación Médica, 8(2), 74-82. Recuperado de http://scielo.isciii.es/pdf/edu/ v8n2/original1.pdf

\section{Cómo citar este artículo en APA:}

Garbanzo, G. M. (enero-abril, 2014). Factores asociados al rendimiento académico tomando en cuenta el nivel socioeconómico: Estudio de regresión múltiple en estudiantes universitarios Revista Electrónica Educare, 18(1), 119-154. Recuperado de http://www.revistas.una.ac.cr/index.php/EDUCARE/issue/current

Nota: Para citar este artículo en otros sistemas puede consultar el hipervínculo "Como citar el artículo" en la barra derecha de nuestro sitio web:

http://www.revistas.una.ac.cr/index.php/EDUCARE/index 